\title{
OS3D/GIMRT . \\ Software for Modeling Multicomponent-Multidimensional Reactive Transport
}

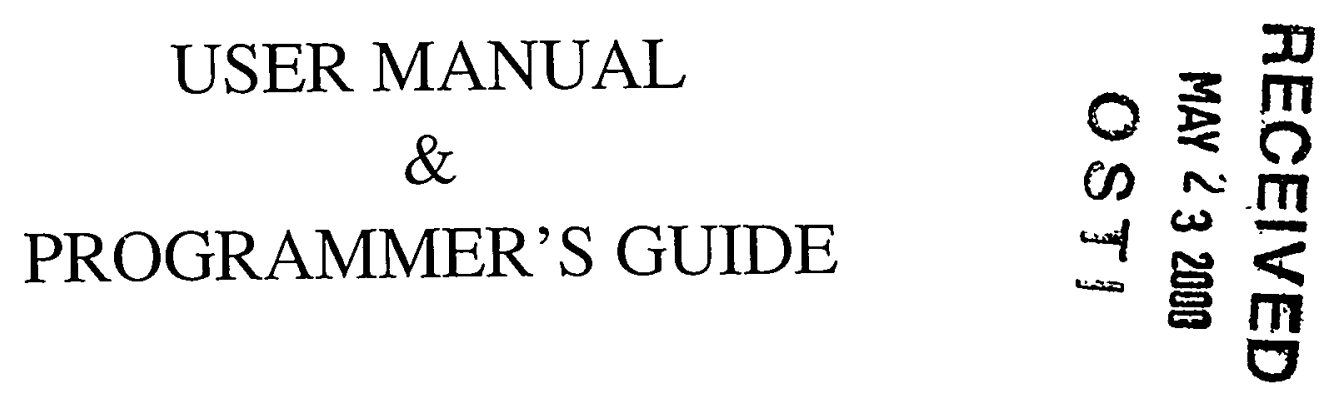

\section{I. Steefel ${ }^{1}$ S.B. Yabusaki}

Pacific Northwest National Laboratory

Richland, Washington 99352

U.S.A.

1) Department of Geology, University of South Florida Tampa, Florida 33620 U.S.A.

E-mail: steefel@margaux.cas.usf.edu

Copyright (C)1995, 1996 by Battelle Memorial Institute All Rights Reserved

Version 1.0

May 6, 1996 


\section{DISCLAIMER}

This report was prepared as an account of work sponsored by an agency of the United States Government. Neither the United States Government nor any agency thereof, nor any of their employees, make any warranty, express or implied, or assumes any legal liability or responsibility for the accuracy, completeness, or usefulness of any information, apparatus, product, or process disclosed, or represents that its use would not infringe privately owned rights. Reference herein to any specific commercial product, process, or service by trade name, trademark, manufacturer, or otherwise does not necessarily constitute or imply its endorsement, recommendation, or favoring by the United States Government or any agency thereof. The views and opinions of authors expressed herein do not necessarily state or reflect those of the United States Government or any agency thereof. 


\section{DISCLAIMER}

Portions of this document may be illegible in electronic image products. Images are produced from the best available original document. 
OS3D/GIMRT IS PROVIDED "AS IS" AND WITHOUT ANY WARRANTY EXPRESS OR IMPLIED. THE USER ASSUMES ALL RISKS OF USING OS3D/GIMRT. THERE IS NO CLAIM OF THE MERCHANTABILITY OR FITNESS FOR A PARTICULAR PURPOSE.

YOU MAY MODIFY THE SOURCE CODE FOR YOUR OWN USE, BUT YOU MAY NOT DISTRIBUTE EITHER THE ORIGINAL OR THE MODIFIED CODE TO USERS AT ANY SITES OTHER THAN YOUR OWN. 


\section{Contents}

LIST OF CONTENTS $\ldots \ldots \ldots \ldots \ldots \ldots \ldots \ldots \ldots$

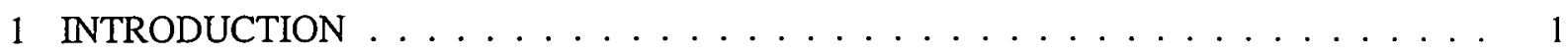

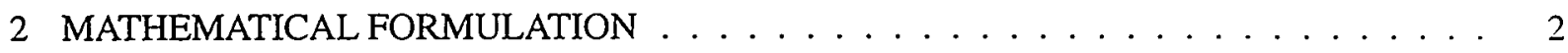

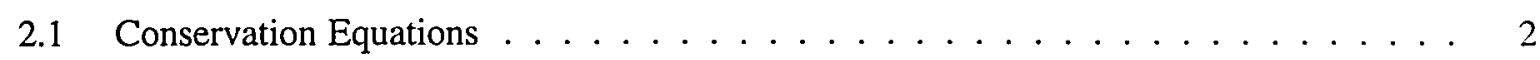

2.2 Example of a Total Concentration $\ldots \ldots \ldots \ldots \ldots$

2.3 Reaction Rate Laws . . . . . . . . . . . . . . . 5

2.4 Example involving Albite Dissolution . . . . . . . . . . . . . 7

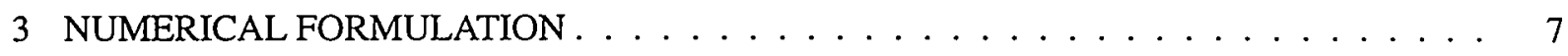

3.1 Global Implicit Approach $\ldots \ldots \ldots \ldots \ldots$

3.2 Operator Splitting/Sequential Iteration Approach $\ldots \ldots \ldots$

3.3 Integrated Finite Difference Formulation . . . . . . . . . . . . . . 10

3.4 Transport Algorithm in Global Implicit Approach . . . . . . . . . . . . . . 12

3.5 Transport Algorithm in Operator Splitting Approach . . . . . . . . . . . 13

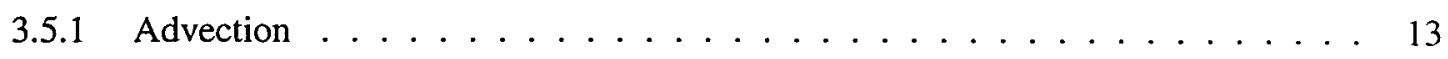

3.5.2 Diffusion and Dispersion . . . . . . . . . . . . . . 14

3.6 Solving the Equations with Newton's Method . . . . . . . . . . . . . . 14

3.7 Structure of the Jacobian Matrix . . . . . . . . . . . . . 16

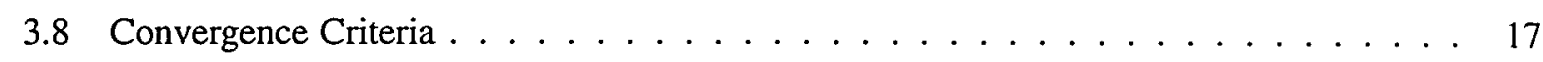

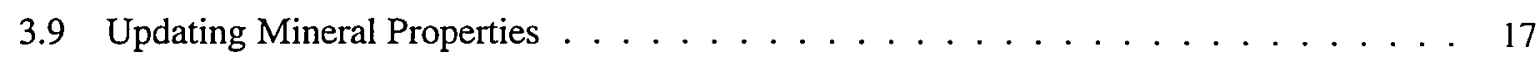

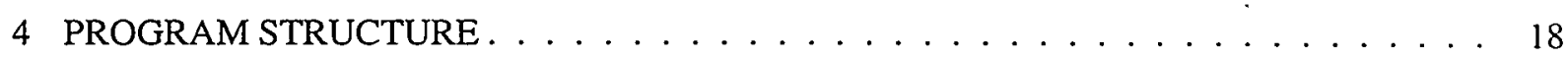

4.1 Global Implicit . . . . . . . . . . . . . . . . . 18

5 FEATURES OF THE CODE $\ldots \ldots \ldots \ldots \ldots \ldots \ldots$

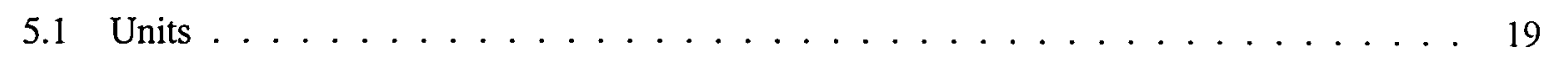

5.2 Boundary Conditions . . . . . . . . . . . . . . . . 19

5.3 Choice of Primary and Secondary Species $\ldots \ldots \ldots \ldots \ldots$

5.4 Redox Reactions . . . . . . . . . . . . . . . . . . . 23 
5.5 Acid-Base Reactions . . . . . . . . . . . . . . . . . . . . . . 24

5.6 Basis Switching . . . . . . . . . . . . . . . . . . . 24

5.7 Activity Coefficient Model . . . . . . . . . . . . . . . . . . 24

5.8 Initialization Procedure . . . . . . . . . . . . . . . . . 24

5.9 Temperature Gradients . . . . . . . . . . . . . . . . 25

5.10 Updating Porosity and the Transport Coefficients $\ldots \ldots \ldots 26$

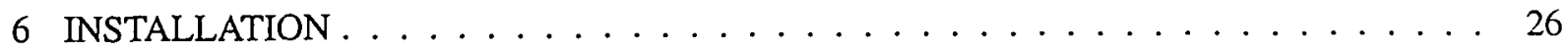

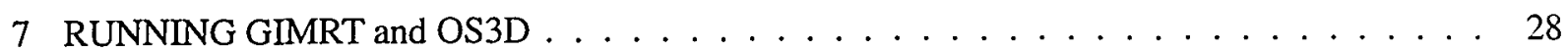

7.1 Control Parameters . . . . . . . . . . . . . . . . 28

7.2 Specifying Primary and Secondary Species $\ldots \ldots \ldots$

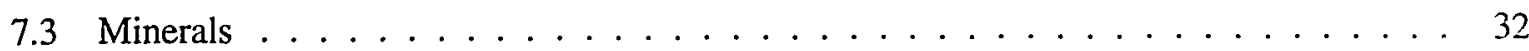

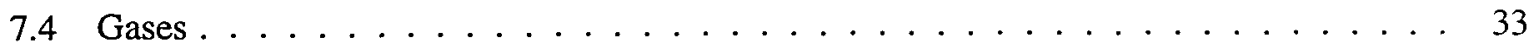

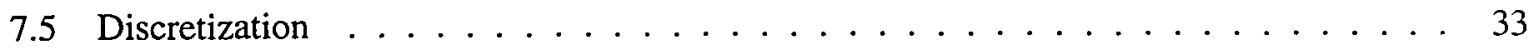

7.6 Geochemical Conditions . . . . . . . . . . . . . . . . 34

7.7 Spatial Distribution of Geochemical Conditions $\ldots \ldots \ldots$

7.8 Reaction Path Switch and Plotting Information . . . . . . . . . . . . . 39

7.9 Transport Parameters . . . . . . . . . . . . . . . . . . . . . 39

8 PARAMETERS IN FORTRAN FILES . . . . . . . . . . . . . . . . . 41

9 OUTPUT IN THE FILE THREEDOUT $\ldots \ldots \ldots \ldots \ldots \ldots$

10 GRAPHICS OUTPUT $\ldots \ldots \ldots \ldots \ldots \ldots \ldots$

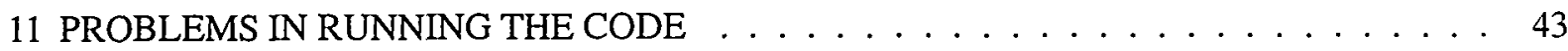

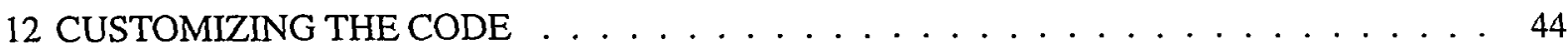

12.1 Array Dimensions . . . . . . . . . . . . . . . . . . 44

12.2 Modifying the Data Base . . . . . . . . . . . . . . . . . 44

12.3 Adding or Modifying Kinetic Rate Laws . . . . . . . . . . . . . . 45

12.4 Providing and/or Updating a Velocity Field . . . . . . . . . . . . . . 45

12.5 Adding a Temperature Field $\ldots \ldots \ldots \ldots$ 
13 ACKNOWLEDGMENTS $\ldots \ldots \ldots \ldots \ldots \ldots \ldots \ldots$

14 REFERENCES . . . . . . . . . . . . . . . . . . . . . . . . 49

15 DESCRIPTION OF THE INPUT FILE THREEDIN $\ldots \ldots \ldots \ldots$

\section{List of Tables}

1 Sample data base read by GIMRT and OS3D for a single temperature of $25^{\circ} \mathrm{C}$. . . . 47

\section{List of Figures}

1 Discretization scheme for a one-dimensional problem using integrated finite differences. 11

2 Program structure for GIMRT which uses a global implicit or one-step method used to solve the multi-component reaction-transport equation. . . . . . . . . . . . 20

3 Program structure for OS3D which uses time splitting of the transport and reaction step or a sequential iteration between transport and reaction. . . . . . . . . . . 21 


\section{INTRODUCTION}

OS3D/GIMRT is a numerical software package for simulating multicomponent reactive transport in porous media. The package consists of two principal components: 1) the code OS3D (Operator Splitting 3Dimensional Reactive Transport) which simulates reactive transport by either splitting the reaction and transport steps in time (i.e., the classic time or operator splitting approach) or by iterating sequentially between reactions and transport (e.g., Yeh and Tripathi, 1989: Walter et al., 1994), and 2) the code GIMRT (Global Implicit Multicomponent Reactive Transport) which treats up to two dimensional reactive transport with a one step or global implicit approach (Steefel and Lasaga, 1994). Although the two codes do not yet have totally identical capabilities, they can be run from the same input file, allowing comparisons to be made between the two approaches in many cases. The advantages and disadvantages of the two approaches are discussed more fully below, but in general, OS3D is designed for simulation of transient concentration fronts, particularly under high Peclet number transport conditions, because of its use of a total variation diminishing or TVD transport algorithm. GIMRT is suited for simulating water-rock alteration over long periods of time where the aqueous concentration field is at or close to a quasi-stationary state and the numerical transport errors are less important. Where water-rock interaction occurs over geological periods of time, GIMRT may be preferable to OS3D because of its ability to take larger time steps..

Both OS3D and GIMRT are based on a continuum representation of transport and reaction in porous media. The codes solve the nonlinear set of partial differential equations describing coupled reaction and transport in a multicomponent system by using finite difference techniques. The finite difference method, although computationally more intensive than the simpler reaction path models, allows for modeling of the entire range of both transient and steady-state phenomena, including advective, dispersive, and diffusive transport or any combination of these. A second important feature of the codes is the use of a kinetic formulation for all mineral dissolution and precipitation reactions. This allows one to avoid a priori assumptions of local equilibrium, although it is still possible to obtain local equilibrium behavior where the rates of reaction are much greater than the rates of mass transport. In contrast, aqueous and surface complexation reactions (including redox) are assumed to be reversible at this time, although it is planned that this requirement will be relaxed in future developments.

The package OS3D/GIMRT includes a number of notable features. OS3D can be run in OD ("D" here refers to the spatial dimensions, with OD being a reaction path or batch reaction calculation), $1 \mathrm{D}, 2 \mathrm{D}$, or 3D simply by changing the input file. GIMRT possesses the same flexibility but is currently limited to two spatial dimensions. At startup, the codes allow one to specify a number of different zones within the domain of interest which have distinct initial solute concentrations, mineral abundances, and/or grid spacing. The code also includes a distribution of species calculation to specify the initial and boundary solute concentrations. The code reads a thermodynamic data base based on the EQ3/EQ6 (Wolery and others, 1990) data base which covers temperatures between 0 and $300^{\circ} \mathrm{C}$ along the water saturation curve for a large number of mineral and aqueous species. The user can simulate reactive transport at higher pressures by providing the appropriate thermodynamic data from, for example, the code SUPCRT. The code also incorporates automatic basis switching (rewriting the reactions in terms of the dominant species) which can considerably improve the convergence behavior in some cases (particularly redox), or it can be run using a constant set of primary or basis species specified initially by the user. The codes also allow for a range of mineral-water reaction rate laws, including parallel reactions (e.g., $\mathrm{pH}$-dependent and $\mathrm{pH}$ independent rates) and proton-promoted and/or ligand-promoted dissolution. At the present time, only GIMRT can treat non-isothermal conditions.

Neither code currently has a hydrodynamic flow calculation coupled directly to it. At the present time, therefore, one must provide a velocity field calculated externally. A fully coupled flow code, with options 
for porosity and permeability change as a function of time, will be included in the next release.

\section{MATHEMATICAL FORMULATION}

\subsection{Conservation Equations}

The governing equation for the conservation of solute mass is given by (Steefel, 1992; Steefel and Lasaga, 1994)

$$
\frac{\partial}{\partial t}\left(\phi \rho_{f} M_{H_{2} O} C_{\imath}\right)+\nabla \bullet\left(-\mathrm{D} \nabla\left(\rho_{f} M_{H_{2} O} C_{\imath}\right)+\mathrm{u} \rho_{f} M_{H_{2} O} C_{\imath}\right)=R_{\imath} \quad\left(\imath=1,2, \ldots N_{t o t}\right),
$$

where $C_{2}$ is the molal concentration of a species in solution (in units of moles per $\mathrm{kg} \mathrm{H}_{2} \mathrm{O}$ ), $M_{\mathrm{H}_{2} \mathrm{O}}$ is the . mass fraction of $\mathrm{H}_{2} \mathrm{O}, \rho_{f}$ is the fluid density, $\mathbf{u}$ is the Darcian flux, $\mathbf{D}$ is the combined dispersion-diffusion tensor, $R_{\imath}$ (in units of moles per unit volume rock per unit time) is the total reaction rate of species $\imath$ in solution, and $N_{t o t}$ is the total number of aqueous species. Note that the porosity, $\phi$, is imbedded in both the flux terms and the reaction terms as described below.

The reaction term $R_{\imath}$ is divided into dissolution-precipitation (heterogeneous) reactions, $R_{\imath}^{\text {min }}$, aqueous (homogeneous) reactions, $R_{2}^{a q}$, and adsorption reactions, $R_{2}^{a d s}$, such that

$$
R_{\imath}=R_{\imath}^{\min }+R_{\imath}^{a q}+R_{\imath}^{a d s}
$$

The reaction rate is written here in terms of a unit volume of rock but could be written equivalently per unit volume of fluid if it were then multiplied by the porosity, $\phi$. The Darcian flux is related to the true velocity of the fluid, $v$, by

$$
\mathrm{u}=\phi \mathbf{v}
$$

D is a second order tensor which is defined as the sum of the mechanical or kinematic dispersion coefficient $\mathrm{D}^{*}$ and the molecular diffusion coefficient, $D_{0}$, in water divided by the formation resistivity factor, $F$

$$
\mathrm{D}=\mathrm{D}^{*}+\frac{D_{0}}{F}
$$

$F$ is defined as the ratio of the resistivity of the saturated porous medium over the resistivity of the pore solution alone (Marsily, 1986). The resistivity factor, $F$, may be defined in numerous ways (see, for example, Dullien, 1979), but here a definition based on Archie's Law is used which gives the formation factor as

$$
F=\phi^{-m}
$$

where $m$ is the "cementation exponent". Dullien (1979) reports values of $m$ which range between about 1.3 and 2.5. The kinematic dispersion coefficient is written here as

$$
D_{i i}^{*}=\alpha_{T}|u|+\left(\alpha_{L}-\alpha_{T}\right) \frac{u_{i}^{2}}{|u|}
$$

where $\alpha_{L}$ and $\alpha_{T}$ are the longitudinal and transverse dispersivities respectively (Bear, 1979; Marsily, 1986) and $|u|$ is the magnitude of the velocity. In both OS3D and GIMRT, off-diagonal dispersion coefficients (i.e., $D_{i j}^{*}$, where $i$ and $j$ are coordinate directions) are not included at the present time. 
The heterogeneous reaction term $R_{\imath}^{\min }$ can be written as the sum of all the individual mineral-water reactions which affect the concentration of the $\imath$ th species

$$
R_{\imath}^{m i n}=-\sum_{m=1}^{N_{m}} \nu_{\imath m} r_{m}
$$

where $r_{m}$ is the rate of precipitation or dissolution of mineral $m$ per unit volume rock, $\nu_{2 m}$ is the number of moles of $\imath$ in mineral $m$ (or stoichiometric coefficient if the reaction is written in terms of the dissolution of one mole of the mineral), and $N_{m}$ is the number of minerals present in the rock. Following convention, we take $r_{m}$ as positive for precipitation and negative for dissolution.

Equation 1 provides a general formulation for the conservation of solute mass which makes no assumptions of chemical equilibrium. If we assume that the various aqueous species are in chemical equilibrium, however, it is possible to reduce the number of independent concentrations, that is, the number that actually need to be solved for. Mathematically, this means that in a system containing $N_{\text {tot }}$ aqueous species, the number of independent chemical components in the system $N_{c}$ is reduced from the total number of species by the $N_{x}$ linearly independent chemical reactions between them (for further discussion, see Hooyman, 1961; Aris, 1965; Bowen, 1968; Van Zeggeren and Storey, 1970; Reed, 1982; Lichtner, 1985; Kirkner and Reeves, 1988). This leads to a natural partitioning of the system into $N_{c}$ primary or $b a$ sis species, designated here as $C_{\jmath}$, and the $N_{x}$ secondary species, referred to as $X_{\imath}$ (Reed, 1982; Lichtner, 1985; Kirkner and Reeves, 1988). The equilibrium chemical reactions between the primary and secondary species take the form

$$
A_{\imath} \rightleftharpoons \sum_{j=1}^{N_{c}} \nu_{\imath \jmath} A_{\jmath} \quad\left(\imath=1, \ldots, N_{x}\right)
$$

where the $A_{3}$ and the $A_{2}$ are the chemical formulas of the primary and secondary species respectively and $\nu_{\imath \jmath}$ is the number of moles of primary species $\jmath$ in one mole of secondary species $\imath$. It should be noted here that the partitioning between the primary and secondary species is not unique, that is, we can write the chemical reactions in more than one way. The reversible reactions provide an algebraic link between the primary and secondary species via the law of mass action for each reaction

$$
X_{\imath}=K_{2}^{-1} \gamma_{\imath}^{-1} \prod_{\jmath=1}^{N_{c}}\left(\gamma_{\jmath} C_{\jmath}\right)^{\nu_{\imath \jmath}} \quad\left(\imath=1, \ldots, N_{x}\right),
$$

where $\gamma_{3}$ and $\gamma_{2}$ are the activity coefficients for the primary and secondary species respectively, and the $K_{\imath}$ are the equilibrium constants of the reaction given in Equation 8, written here as the destruction of one mole of the secondary species. Equation 8 implies that the rate of production of a primary component $J$ due to homogeneous reactions can be written in terms of the sum of the total rates of production of the secondary species (Kirkner and Reeves, 1988)

$$
R_{\jmath}^{a q}=-\sum_{\imath=1}^{N_{x}} \nu_{\imath \jmath} r_{\imath}
$$

where $r_{2}$ are the reaction rates of the secondary species. Equation 10 suggests that one can think of a mineral dissolving, for example, as producing only primary species which then equilibrate instantly with the secondary species in the system. Using Equation 10, the rates of the equilibrium reactions can be eliminated (Lichtner, 1985; Kirkner and Reeves, 1988)

$$
\begin{gathered}
\frac{\partial}{\partial t}\left[\phi \rho_{f} M_{H_{2} O}\left(C_{\jmath}+\sum_{\imath=1}^{N_{x}} \nu_{\imath \jmath} X_{\imath}\right)\right]+ \\
\nabla \bullet\left[u \rho_{f} M_{H_{2} O}\left(C_{\jmath}+\sum_{\imath=1}^{N_{x}} \nu_{\imath \jmath} X_{2}\right)-D \nabla\left\{\rho_{f} M_{H_{2} O}\left(C_{\jmath}+\sum_{\imath=1}^{N_{x}} \nu_{\imath \jmath} X_{\imath}\right)\right\}\right] \\
=R_{\jmath}^{\min } \quad\left(\jmath=1, \ldots, N_{c}\right)
\end{gathered}
$$


Note that only the term $R_{j}^{\text {min }}$ remains on the right hand side of Equation 11 because we have assumed that they are the only kinetic reactions.

\subsection{Example of a Total Concentration}

If a total concentration, $U_{\jmath}$, is defined (Reed, 1982; Lichtner, 1985; Kirkner and Reeves, 1988)

$$
U_{\jmath}=C_{\jmath}+\sum_{\imath=1}^{N_{x}} \nu_{\imath \jmath} X_{\imath}
$$

then the governing differential equations can be written in terms of the total concentrations in the case where only aqueous (and therefore mobile) species are involved (Kirkner and Reeves, 1988)

$$
\frac{\partial}{\partial t}\left(\phi \rho_{f} M_{H_{2} O} U_{\jmath}\right)+\nabla \bullet\left(u \rho_{f} M_{H_{2} O} U_{\jmath}-D \nabla\left(\rho_{f} M_{H_{2} O} U_{\jmath}\right)\right)=R_{\jmath}^{\min } \quad\left(\jmath=1, \ldots, N_{c}\right) .
$$

As pointed out by Reed (1982) and Lichtner (1985), the total concentrations can usually be interpreted in a straightforward fashion as the total elemental concentrations (e.g., total aluminum in solution), but in the case of $\mathrm{H}^{+}$and redox species, the total concentration has no simple physical meaning and the total concentrations may take on negative values. Where the system includes sorbed species (i.e., surface complexes) which are immobile, then it is a necessary to define both a mobile total concentration

$$
U_{j}^{\text {mobile }}=C_{j}^{\text {mobile }}+\sum_{\imath=1}^{N_{x}} \nu_{\imath \jmath} X_{\imath}^{\text {mobile }},
$$

and an immobile total concentration made up of the surface complexes

$$
U_{\jmath}^{\text {immobile }}=C_{\jmath}^{\text {immobile }}+\sum_{\imath=1}^{N_{x}} \nu_{\imath \jmath} X_{\imath}^{i m m o b i l e}, .
$$

The governing differential equation then becomes

$$
\begin{gathered}
\frac{\partial}{\partial t}\left(\phi \rho_{f} M_{H_{2} O}\left(U_{\jmath}^{\text {mobile }}+U_{\jmath}^{\text {immobile }}\right)+\right. \\
\nabla \cdot\left(u \rho_{f} M_{H_{2} O} U_{\jmath}^{\text {mobile }}-D \nabla\left(\rho_{f} M_{H_{2} O} U_{\jmath}^{\text {mobile }}\right)\right)=+R_{\jmath}^{\text {min }} \quad\left(\jmath=1, \ldots, N_{c}\right) .
\end{gathered}
$$

The concept of a total concentration will be clarified with a few examples. Consider a system in which the aqueous species consist of $\mathrm{H}_{2} \mathrm{O}, \mathrm{H}^{+}, \mathrm{OH}^{-}, \mathrm{Al}^{+++}$, and $\mathrm{Al}(\mathrm{OH})_{4}^{-}$. Assuming that these species are all in equilibrium, then the number of unknown concentrations (i.e., $N_{c}$ ) is reduced from the total number of species concentrations by the number of independent equilibrium reactions $\left(N_{x}\right)$ between them

$$
\begin{gathered}
\mathrm{Al}^{+++}+4 \mathrm{H}_{2} \mathrm{O} \rightleftharpoons \mathrm{Al}(\mathrm{OH})_{4}^{-}+4 \mathrm{H}^{+} \\
\mathrm{H}^{+}+\mathrm{OH}^{-} \rightleftharpoons \mathrm{H}_{2} \mathrm{O}
\end{gathered}
$$

Choosing $\mathrm{H}_{2} \mathrm{O}, \mathrm{H}^{+}$, and $\mathrm{Al}^{+++}$as the primary species and writing the chemical reactions in matrix form as in Equation 8 (that is, with the production or destruction of one mole of the secondary species), the reactions become

$$
\left[\begin{array}{lll}
4 & -4 & 1 \\
1 & -1 & 0
\end{array}\right]\left[\begin{array}{c}
\mathrm{H}_{2} \mathrm{O} \\
H^{+} \\
A l^{+++}
\end{array}\right]=\left[\begin{array}{c}
A l(O H)_{4}^{-} \\
O H^{-}
\end{array}\right]
$$


which implies that the total concentrations are

$$
\begin{gathered}
U_{\mathrm{H}_{2} \mathrm{O}}=C_{\mathrm{H}_{2} \mathrm{O}}+4 C_{\mathrm{Al}(\mathrm{OH})_{4}^{-}}+C_{\mathrm{OH}^{-}} \\
U_{\mathrm{H}^{+}}=C_{\mathrm{H}^{+}}-4 C_{\mathrm{Al}(\mathrm{OH})_{4}^{-}}-C_{\mathrm{OH}^{-}} \\
U_{\mathrm{Al}+++}=C_{\mathrm{Al}+++}+C_{\mathrm{Al}(\mathrm{OH})_{4}^{-}}
\end{gathered}
$$

One can think of the total concentration, then, as a linear combination of the concentration of the primary or basis species and the amount of that primary species present in the secondary species. The $U_{j}$ 's, therefore, account for all of the solute mass in the system, even though in certain cases (e.g., at high $\mathrm{pH}$ where the concentration of $\mathrm{OH}^{-}$is greater than the concentration of $\mathrm{H}^{+}$), the total concentration of $\mathrm{H}^{+}$may be negative.

As noted above, the choice of $\mathrm{H}_{2} \mathrm{O}, \mathrm{H}^{+}$, and $\mathrm{Al}^{+++}$as the primary species is not unique and it is therefore possible to choose a different set of primary species which is mathematically equivalent. If we choose to make $\mathrm{Al}(\mathrm{OH})_{4}^{-}$the primary species, then the stoichiometric reaction matrix becomes

$$
\left[\begin{array}{ccc}
-4 & 4 & 1 \\
1 & -1 & 0
\end{array}\right]\left[\begin{array}{c}
\mathrm{H}_{2} \mathrm{O} \\
H^{+} \\
A l(\mathrm{OH})_{4}^{-}
\end{array}\right]=\left[\begin{array}{c}
A l^{+++} \\
O H^{-}
\end{array}\right]
$$

so that the total concentrations now become

$$
\begin{gathered}
U_{\mathrm{H}_{2} \mathrm{O}}=C_{\mathrm{H}_{2} \mathrm{O}}-4 C_{\mathrm{Al}^{+++}}+C_{\mathrm{OH}^{-}} \\
U_{\mathrm{H}^{+}}=C_{H^{+}}+4 C_{\mathrm{Al}^{+++}}-C_{\mathrm{OH}^{-}} \\
U_{\mathrm{Al}(\mathrm{OH})_{4}^{-}}=C_{\mathrm{Al}(\mathrm{OH})_{4}^{-}}+C_{\mathrm{Al}^{+++}}
\end{gathered}
$$

Writing the chemical reactions differently (which mathematically is referred to as a change of basis), therefore, changes the total concentrations of $\mathrm{H}_{2} \mathrm{O}$ and $\mathrm{H}^{+}$, even though the total concentration of the Al-bearing species is the same. In the codes OS3D and GIMRT, the user has the option of either leaving basis set always the same or of dynamically calculating a new basis set at each grid point in space so that the basis species chosen is the one with the largest concentration. The basis switching makes for a more diagonally dominant Jacobian matrix and therefore may improve the convergence behavior of the algorithm.

\subsection{Reaction Rate Laws}

The codes OS3D and GIMRT use a kinetic rate law based on the assumption that attachment and detachment of ions from mineral surfaces is the rate-limiting step (Steefel and Lasaga, 1994). The principal computational advantage of this approach is that there are no additional concentrations of ions immediately adjacent to the mineral surfaces to be solved for as there are when diffusion to and from the surface is rate-limiting (Steefel, 1992; Steefel and Lasaga, 1994). Where surface attachment is the rate-limiting step, the concentrations next to the mineral's surface are just the concentrations of those ions in the bulk solution which we are already solving for. If the mineral volume fractions are held constant for a single time step and updated only after convergence of the aqueous and surface species, then we can solve for 
the minimum number of equations, which is $N_{c} \times M$ where $N_{c}$ is the number of independent chemical components and $M$ is the number of grid points in the system.

The rate laws used for mineral precipitation and dissolution in 1DREACT are based loosely on transition state theory (e.g., Lasaga, 1981; Aagaard and Helgeson, 1982; Lasaga, 1984). The formulation gives the dependence of the rate on the saturation state of the solution with respect to a particular mineral as a function of the ion activity product, $Q_{m}$, defined by

$$
Q_{m}=\prod_{\jmath=1}^{N_{c}} a_{\jmath}^{\nu_{m}}
$$

where the $a_{\jmath}$ are the activities of the ions making up the mineral and the $\nu_{m j}$ are the stoichiometric coefficients. The following is the form for the rate law of crystal growth and dissolution of a mineral (Lasaga, 1984; Steefel and Van Cappellen, 1990; Steefel and Lasaga, 1994)

$$
r_{m}=\operatorname{sgn}\left(\log \left[Q_{m} / K_{m}\right]\right) A_{m} k_{m}\left(\prod_{\imath=1}^{N_{c}+N_{x}} a_{\imath}^{p}\right)\left|\left(\frac{Q_{m}}{K_{m}}\right)^{\mathcal{M}}-1\right|^{n}
$$

where $k$ is either the growth or dissolution rate constant, $a_{2}$ is the activity of a species raised to an empirically determined power $p$ which affects the far from equilibrium dissolution rate (e.g., $\left.\mathrm{H}^{+}\right), A_{m}$ is the surface area of the reacting mineral, and $\mathcal{M}$ and $n$ are two positive numbers which are also normally determined by experiment. The bars $\mid$ | refer to the absolute value of the quantity and the term $\operatorname{sgn}\left(\log Q_{m} / K_{m}\right)$ gives the sign of the expression, negative if the fluid is undersaturated and positive if the fluid is supersaturated with respect to the mineral. This formulation ensures that the reaction rate, $r_{m}$, has the correct sign when $n \neq 1 . K_{m}$ is the equilibrium constant for the mineral-water reaction written as the destruction of one mole of mineral $m$.

In the input file containing the mineral-fluid rate data, the user has a choice of specifying the activities of those species which will affect the far from equilibrium dissolution rate. In the case where no species are given, the far from equilibrium dissolution rate becomes zeroth order, since $Q_{m} / K_{m}$ is a small number under these circumstances. The user, however, may provide a dependence on any of the either primary or secondary species concentration in solution. In addition, multiple parallel reactions may be specified. This allows one to easily incorporate, for example, both a $\mathrm{pH}$-independent and a $\mathrm{pH}$-dependent (i.e., proton-promoted) rate of dissolution for a mineral which are then summed to give the total dissolution rate. One can also model ligand-promoted dissolution with this scheme by specifying that the far from equilibrium rate be proportional to the concentration of reactive adsorbed ligand. Several examples are given below.

The temperature dependence of the reaction rate constant can be expressed reasonably well via an Arrhenius equation (Lasaga, 1984). Since many rate constants are reported at $25^{\circ} \mathrm{C}$, it is more convenient to write the rate constant at some temperature as

$$
k=k_{25} \exp \left[\frac{-E_{a}}{R}\left(\frac{1}{T}-\frac{1}{298.15}\right)\right]
$$

where $E_{a}$ is the activation energy, $k_{25}$ is the rate constant at $25^{\circ} \mathrm{C}, R$ is the gas constant, and $T$ is temperature in the Kelvin scale. Rate constants at temperature can be computed by providing the appropriate activation energy and the rate at $25^{\circ} \mathrm{C}$, or by inputting the rate constant at temperature and specifying a zero activation energy. 


\subsection{Example involving Albite Dissolution}

In this section, we give an example involving albite dissolution to show how both the aqueous complexation reactions and the mineral reaction rates result in a nonlinear system. Here we consider only the ODEs which result from the reaction term (i.e., the equations would be those in the reaction module when using either operator splitting or sequential iteration). If a global implicit approach were used, one would also have terms involving spatial derivatives in the equation. If we consider a geochemical system involving the primary species $\mathrm{H}_{2} \mathrm{O}, \mathrm{H}^{+}, \mathrm{Al}^{+3}, \mathrm{SiO}_{2(a q)}$, and $\mathrm{Na}^{+}$and the secondary species (all in equilibrium with the primary species) $\mathrm{OH}^{-}, \mathrm{AlOH}_{4}^{-}, \mathrm{H}_{3} \mathrm{SiO}_{4}^{-}$, and $\mathrm{NaCl}_{(a q)}$, then the differential equations take the form

$$
\begin{gathered}
\frac{\mathrm{d}\left(N a^{+}+N a C l_{(a q)}\right)}{\mathrm{d} t}=A_{a l b} a_{H^{+}}^{0.4} k_{a l b}\left[1-\left(\frac{a_{N a^{+}} a_{A l+3} a_{S i o_{2(a q)}}^{3}}{a_{H^{+}}^{4}}\right) K_{a l b}^{-1}\right], \\
\frac{\mathrm{d}\left(S_{i o_{2(a q)}}+H_{3} S i O_{4}^{-}\right)}{\mathrm{d} t}=3 A_{a l b} a_{H^{+}}^{0.4} k_{a l b}\left[1-\left(\frac{\left.a_{N a^{+} a_{A l^{+3}} a_{S i o_{2(a q)}}^{3}}\right)}{a_{H^{+}}^{4}}\right) K_{a l b}^{-1}\right], \\
\frac{\mathrm{d}\left(A l^{+3}+A l O H_{4}^{-}\right)}{\mathrm{d} t}=A_{a l b} a_{H^{+}}^{0.4} k_{a l b}\left[1-\left(\frac{\left.\left.a_{N a^{+} a_{A l^{+3}} a_{S i o_{2(a q)}}^{3}}\right) K_{a l b}^{-1}\right]}{a_{H^{+}}^{4}}\right)\right. \\
\frac{\mathrm{d}\left(H^{+}-O H^{-}-4 A l O H_{4}^{-}-H_{3} S i O_{4}^{-}\right)}{\mathrm{d} t}=-4 A_{a l b} a_{H^{+}}^{0.4} k_{a l b}\left[1-\left(\frac{a_{N a^{+} a_{A l}+3} a_{S i o_{2(a q)}}^{3}}{a_{H^{+}}^{4}}\right) K_{a l b}^{-1}\right],
\end{gathered}
$$

where we have neglected the mass balance of $\mathrm{H}_{2} \mathrm{O}$. The actual form of the equations solved by both OS3D and GIMRT eliminates the secondary species entirely, writing each in terms of the primary species using Equation 9.

\section{NUMERICAL FORMULATION}

GIMRT uses a global implicit or one-step method to solve the reaction-transport equation. In contrast, OS3D uses either classic time splitting of the transport and reaction terms or it can be run with the sequential iteration method proposed by Yeh and Tripathi (1989). There are some advantages to each approach. Yeh and Tripathi (1989) cite the major advantage of the operator splitting or sequential iteration approach as the lower memory requirements of these methods compared to the global implicit and the greater speed with which a single time step can be completed. Although these may be advantages of the time splitting approach over the global implicit, probably the most significant advantage of the time splitting approach is the ability to use algorithms for high Peclet number transport (i.e., advection dominating over dispersion and molecular diffusion) which have less numerical dispersion than those readily usable in a global implicit scheme. GIMRT, for example, using a first-order accurate upwind scheme, which along with the backwards differentiation, leads to a significant amount of numerical dispersion. In contrast, OS3D uses a third order accurate total variation diminishing or TVD method proposed by Gupta (1991). The TVD algorithm results in signficantly less numerical dispersion than the upwind scheme used in the global implicit.

The chief advantage of the global implicit approach, is that unlike the TVD method, one is not restricted rigorously to the Courant condition, which requires that mass not be transported more than a single grid 
cell in any one timestep. Although this is an important criterion to observe in order to maintain accuracy in transient problems, it is less important once the aqueous concentrations approach a quasi-stationary state. In simulations we have carried out, we find that the operator splitting error is minimal when the Courant number is less than about 0.2 , so even if one does not use an explicit transport step, minimizing operator splitting error requires a small timestep. If the interest in the problem is to model water-rock interaction over geological periods of time, then transport errors associated with the transient propagation of concentration fronts are less significant. In these cases, the global implicit approach can offer a real advantage because of the ability to take larger time steps once the system relaxes to a quasi-stationary state. In contrast, stability requirements dictate that the TVD algorithm, which uses an explicit or forward Euler method, use a timestep smaller than a Courant number of approximately $1 / 2$.

\subsection{Global Implicit Approach}

In the one-step method employed by GIMRT, the transport and reaction terms are solved simultaneously. One approach would be to solve directly for the total concentrations (the $U_{j}$ 's) in any one iteration and then follow this with a distribution of species calculation to determine the concentrations of the individual species (see discussion by Kirkner and Reeves, 1988). The method implemented here, however, consists of solving simultaneously for the complexation (which are assumed to be at equilibrium), the heterogeneous reactions, and transport terms. This means that the primary species (the $C_{j}$ 's) rather than the total concentrations are the unknowns. Following the notation of Lichtner (1992), we can introduce the differential operator

$$
\mathcal{L}\left(U_{\jmath}\right)=\left[\frac{\partial}{\partial t} \phi+\nabla \bullet(\mathbf{u}-\mathrm{D} \nabla)\right] U_{\jmath}
$$

if we drop the density and mass fraction of $\mathrm{H}_{2} \mathrm{O}$ terms for simplicity and write the governing differential equations in terms of the total component concentrations as

$$
\mathcal{L}\left(U_{\jmath}\right)=R_{\jmath}^{\min } \quad\left(\jmath=1, \ldots, N_{c}\right)
$$

where the reaction term $R_{j}^{m i n}$ includes only irreversible (in our case, heterogeneous) reactions. Note that in this formulation, we assume that the diffusion coefficients are the same for all of the aqueous species (both primary and secondary). This makes the solution of the reaction-transport equation much simpler because the secondary species do not have to be individually transported.

We proceed by substituting Equation 9 into Equation 11 to obtain an expression for the total soluble concentration in terms of the primary species alone. Substituting this result into Equation 31 gives (essentially a kinetic version of the formulation A of Kirkner and Reeves [1988])

$$
\begin{array}{r}
\mathcal{L}\left[C_{j}+\sum_{i=1}^{N_{x}} \nu_{i j} \gamma_{i}^{-1} K_{i}^{-1} \prod_{j=1}^{N_{c}}\left(\gamma_{j} C_{j}\right)^{\nu_{i j}}\right]+ \\
\sum_{m=1}^{N_{m}} \nu_{i m} \operatorname{sgn}\left(\log \left[Q, K_{m}\right]\right) A_{m} k_{m}\left|\left(\prod_{j=1}^{N_{c}}\left(\gamma_{j} C_{j}\right)^{\nu_{m}} K_{m}^{-1}\right)^{\mathcal{M}}-1\right|^{n}=0 \\
j=1, \cdots N_{c},
\end{array}
$$

where the $\gamma_{2}$ 's and $\gamma_{j}$ 's are the activity coefficients for the secondary and primary species respectively. The integrated finite difference method is used to convert the differential operator $\mathcal{L}$ into an algebraic expression and is discussed in the next section. 


\subsection{Operator Splitting/Sequential Iteration Approach}

Unlike GIMRT, the code OS3D solves the transport terms and the reaction terms separately. Splitting of the transport and reaction steps is presently carried out in OS3D using the Strang scheme, in which a half transport step is followed by a full reaction step which is in turn followed by another half transport step (Strang, 1968; Zysset et al., 1994). This form of time splitting involves a three-step algorithm which requires no iteration and takes the form

$$
\frac{\left(C_{k}^{\text {pre-react }}-C_{k}^{n}\right)}{\Delta t / 2}=L\left(C_{k}\right)^{n}, \quad\left(k=1, \ldots, N_{c}+N_{x}\right),
$$

followed by a reaction step

$$
\frac{\left(U_{j}^{\text {reacted }}\left(c_{j}\right)-U_{j}^{\text {pre-react }}\left(c_{j}\right)\right)}{\Delta t}=R_{j}^{\text {min }} \quad\left(\jmath=1, \ldots, N_{c}\right),
$$

which is in turn followed by another $1 / 2$ transport step

$$
\frac{\left(C_{k}^{n+1}-C_{k}^{r e a c t e d}\right)}{\Delta t / 2}=L\left(C_{k}\right)^{\text {reacted }}, \quad\left(k=1, \ldots, N_{c}+N_{x}\right) .
$$

In this scheme, $C_{k}^{\text {pre-react }}$ are the concentrations of the individual species at the end of the first $1 / 2$ transport step, $U_{k}^{\text {reacted }}$ are the total concentrations at the end of the full reaction step, and $C_{k}^{n+1}$ are the individual species concentrations at the end of the second $1 / 2$ transport step (i.e., at the end of the full reactiontransport time step). $L$ refers to the spatial differential operator defined by

$$
L=[\nabla \cdot(\mathbf{u}-\mathrm{D} \nabla)] .
$$

This scheme is reported to be second order accurate in time (Zysset et al., 1994). Note that in this formulation all of the individual species are transported, while the reaction step is still formulated in terms of the total concentrations which are functions of the individual primary species (i.e., the solution of $N_{c}$ unknowns is required). If basis switching is not used, one could transport the total concentrations in Equation 33 rather than than the individual species concentrations and follow this with a reaction calculation and then another transport of the total concentrations. Where basis switching is used as it often is in the OS3D code, however, it is easier to transport all of the aqueous species so that the algorithm can construct any basis set it chooses from the individually transported species.

The form of the equation solved in the reaction step is the same as in Equation 32, except that in the operator splitting case we do not have the spatial operator, i.e., the operator becomes

$$
\mathcal{L}\left(U_{\jmath}\right)=\left[\frac{\partial}{\partial t} \phi\right] U_{\jmath}
$$

Yeh and Tripathi (1989) and Valocchi and Malmstead (1989) pointed out that in some cases the classic time splitting approach described above can lead to operator splitting error. The global implicit approach, of course, eliminates this error, but at the cost of adding in transport errors associated with the spatial discretization. An alternative is to iterate sequentially between the transport and reaction in order to obtain a solution free from operator splitting error. The sequential iteration method offers a way of doing this while still avoiding the construction and solution of a large global matrix as in the global implicit approach. Our experience, however, is that the sequential iteration scheme offers little improvement over the Strang operating splitting procedure and that it involves considerably more CPU time. At the present 
time, therefore, we recommend using the Strang operator splitting scheme rather than sequential iteration. We have incorporated the sequential iteration approach nontheless and describe it below.

The sequential iteration approach is clearly explained by Walter et al. (1994). In this case, one solves the physical transport equations with a constant reaction term based on species concentrations from the previous iteration. Each transport plus constant reaction term calculation is then followed by a reaction step using the transported and partly reacted concentrations. The algorithm then becomes

$$
\frac{\left(C_{k}^{i n t}-C_{k}^{n}\right)}{\Delta t}=L\left(C_{k}\right)^{n}+R_{k}^{n+1 / 2}, \quad\left(k=1, \ldots, N_{c}+N_{x}\right)
$$

where $R_{k}$ is the total reaction rate for the individual species (i.e., both heterogeneous and homogeneous reactions) and $C_{k}^{\text {int }}$ refers to the transported and partly reacted intermediate concentrations. This is followed by

$$
\frac{\left(U_{\jmath}^{n+1}-U_{\jmath}^{i n t}\right)}{\Delta t / 2}=R_{\jmath}^{\min } \quad\left(\jmath=1, \ldots, N_{c}\right) .
$$

Note that the time step for reaction step is only $1 / 2$ of the full timestep used in the transport step. This results in a reaction term which is centered in time (Walter et al., 1994). The constant reaction term used in Equation 38 is calculated at the end of each reaction step from

$$
R_{k}=\frac{\left(C_{k}^{n+1}-C_{k}^{i n t}\right)}{\Delta t / 2}
$$

Since the term $R_{k}$ used in Equation 38 is only approximate, iteration is required between Equation 38 and Equation 39.

The sequential iteration method often does not change the result obtained using classic time splitting, assuming the Courant number is about 0.2 or less. The user of the code may verify this for themselves with their own application by running OS3D in both modes (procedure described below).

\subsection{Integrated Finite Difference Formulation}

The set of partial differential equations represented by Equation 32 is discretized using the integrated finite difference method (Patankar, 1980; Marsily, 1986). As an example of how the discretization proceeds, consider a set of partial differential equations for the total soluble concentration where the only important transport is via diffusion in one dimension and for simplicity we restrict ourselves to steady state conditions and to a system with constant density. The differential equation is then

$$
\frac{\partial}{\partial x}\left(\phi D \frac{\partial U_{\jmath}}{\partial x}\right)-R_{\jmath}^{m i n}=0
$$

In the integrated finite difference formulation, a control volume is defined and the differential equations are converted to a set of algebraic equations which include the fluxes across the boundaries of the volume and a source or reaction term. Equation 41 can be written (Patankar, 1980)

$$
\left(D \frac{\partial U_{\jmath}}{\partial x}\right)_{e}-\left(D \frac{\partial U_{\jmath}}{\partial x}\right)_{w}-\int_{w}^{e} R_{\jmath}^{m i n} d x=0
$$

where the subscripts $e$ and $w$ are the derivatives evaluated at the two boundaries of the control volume. Equation 42 can be expressed as a set of algebraic equations using piecewise-linear representations of 
the derivatives (Patankar, 1980)

$$
\frac{D_{e}\left(u_{\jmath}^{E}-u_{\jmath}^{P}\right)}{(d x)_{e}}-\frac{D_{w}\left(u_{\jmath}^{P}-u_{\jmath}^{W}\right)}{(d x)_{w}}-R_{\jmath}^{\min } \Delta x=0,
$$

where $D_{e}$ and $D_{w}$ refer to the appropriate value for the diffusion coefficients at the boundary of the volume (averaged between the two grid points) and the $U_{j}$ 's superscripts $(E, W$, and $P$ ) are the total concentrations evaluated at the nodal points themselves (Figure 1). The term $R_{j}^{\min }$ is a function of the primary species at the nodal point $P$. The spacings $(d x)_{e},(d x)_{w}$, and $\Delta x$ are shown in Figure 1.
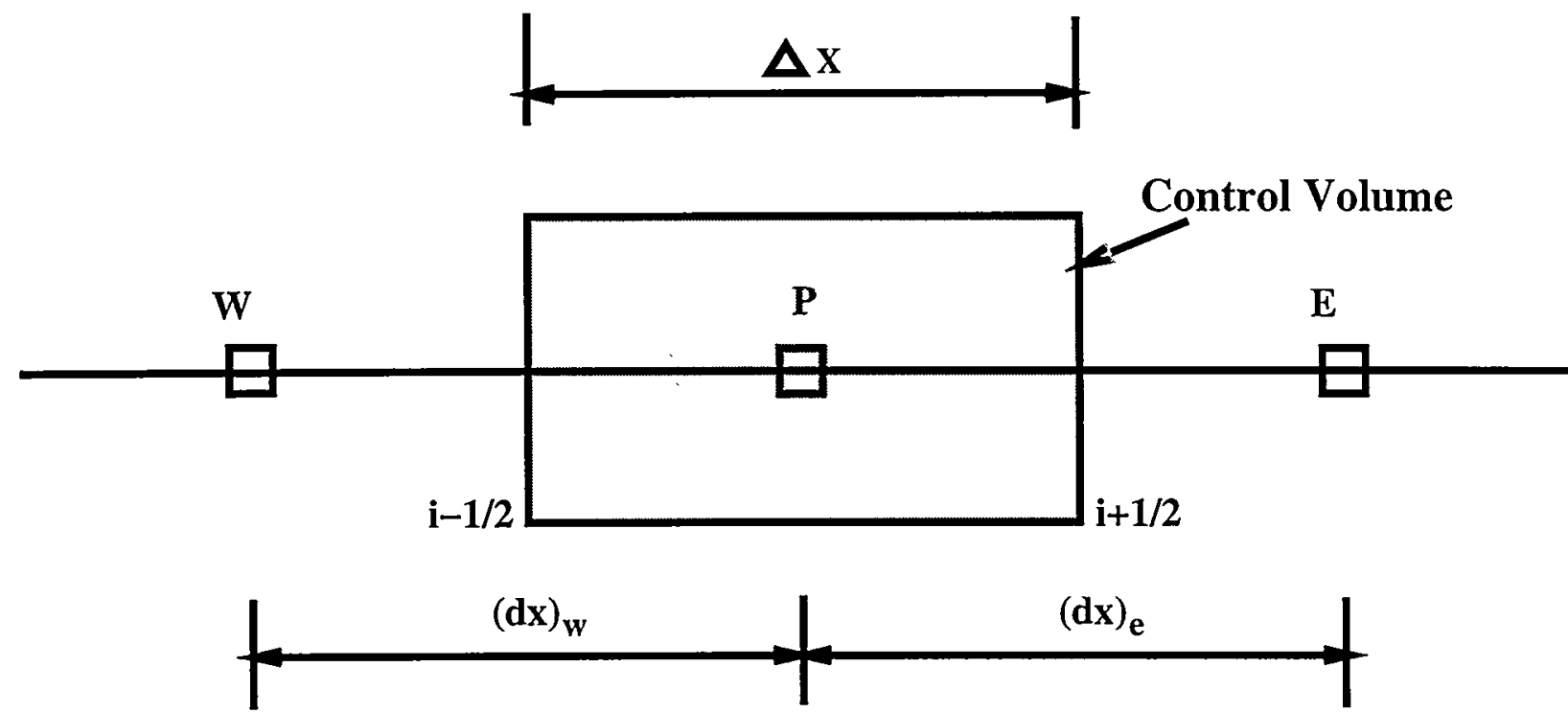

Figure 1: Discretization scheme for a one-dimensional problem using integrated finite differences.

Following the terminology of Patankar (1980), the discretized equations can be recast in the form

$$
a_{P} U_{\jmath}^{P}-a_{E} U_{\jmath}^{E}-a_{W} U_{\jmath}^{W}-d=0
$$

where

$$
\begin{gathered}
a_{E}=\frac{D_{e}}{(d x)_{e}} \\
a_{W}=\frac{D_{w}}{(d x)_{w}} \\
a_{P}=a_{E}+a_{W} \\
d=R_{\jmath}^{\min \Delta x}
\end{gathered}
$$




\subsection{Transport Algorithm in Global Implicit Approach}

The addition of an advection term makes the set of partial differential equations much more difficult to solve stably and accurately (e.g., Press et al., 1986; Patel et al., 1988), although the set of equations can still be cast in the general form of Equation 44. One way to represent the first derivative which appears in the advection term is to use a central difference method

$$
\left(\frac{\partial U}{\partial x}\right)_{P}=\frac{U_{E}-U_{W}}{2 \Delta x}
$$

where the subscript $P$ indicates that the derivative is evaluated at the nodal point $P$ and $\Delta x$ is $1 / 2\left(\delta x_{e}+\right.$ $\left.\delta x_{w}\right)$. The truncation error for the central difference representation is said to be of the order $\Delta x^{2}$ because in the Taylor series expansion upon which it is based, only quadratic terms and higher are neglected (Lapidus and Pinder, 1982). In contrast, a forward difference representation, given by

$$
\left(\frac{\partial U}{\partial x}\right)_{P}=\frac{U_{E}-U_{P}}{(\delta x)_{e}}
$$

has a truncation error of $O(x)$, that is, all terms in the Taylor series higher than first order are truncated. Despite the smaller truncation error of the central difference method, the method may not be numerically stable for advection-dominant problems because of the non-physical oscillations which can occur in the vicinity of sharp concentration fronts (Patankar, 1980; Daus and Frind, 1985; Frind and Germain, 1986; Patel et al, 1988).

It appears, therefore, that the numerical stability of a particular finite difference formulation for the first derivative depends on the relative magnitudes of the advection and dispersion/diffusion terms. The relative importance of the two can be described by the dimensionless grid Peclet number

$$
P e_{\text {grid }}=\frac{v \Delta x}{D}
$$

where $\Delta x$ refers to the grid spacing at any particular point in space. Only for $P e_{\text {grid }}<2$ is the central difference formulation unconditionally stable (Daus and Frind, 1985; Frind and Germain, 1986; Patel et al, 1988). The problem of stability can be "cured" by using a forward difference formulation written in terms of the grid point itself $(P)$ and the upstream or upwind node (Press et al., 1986). It should be pointed out, however, that the upstream-weighted formulation, in addition to having a larger truncation error than the central difference method, achieves its greater stability essentially by adding numerical dispersion (i.e., over and above the physical dispersion present in the problem). In a one-dimensional problem, however, numerical dispersion presents a problem only in tracking transient concentration fronts. The effect does not appear in steady state one-dimensional systems (Patankar, 1980). Numerical dispersion can be reduced by refining the grid in the vicinity of a sharp concentration front.

To take advantage of the greater accuracy of the central difference method at small values of $P e_{\text {grid }}$, a power law scheme proposed by Patankar (1980) is used. This method slides between a fully centered form at $P e_{\text {grid }}<2$ to an upstream-weighted formulation at $P e_{\text {grid }}>10$, where the upwind scheme is necessary for stability. Using the power-law scheme of Patankar (1980), the finite difference coefficients become

$$
\begin{aligned}
& a_{E}=D_{e}\left\|0,\left(1-\frac{0.1 \delta x_{e}\left|v_{e}\right|}{D_{e}}\right)^{5}\right\|+\left\|0,-v_{e}\right\|, \\
& a_{W}=D_{w}\left\|0,\left(1-\frac{0.1 \delta x_{w}\left|v_{w}\right|}{D_{w}}\right)^{5}\right\|+\left\|0, v_{w}\right\|,
\end{aligned}
$$




$$
a_{P}=a_{E}+a_{W}
$$

where the operator $\|A, B\|$ means that the greater of the values $A$ or $B$ is used (equivalent to the FORTRAN operator AMAX1(A,B)) and $|A|$ refers to the absolute magnitude of the value. A fuller description may be found in Patankar (1980).

A fully implicit backward method is used to represent the time derivative (Lapidus and Pinder, 1982). The equations to be solved for each component at each grid point at the $(t+\Delta t)$ time level are then

$$
\frac{\Delta x}{\Delta t}\left(U_{\jmath}^{P}(t+\Delta t)-U_{\jmath}^{P}(t)\right)+a_{P} U_{\jmath}^{P}(t+\Delta t)-a_{E} U_{\jmath}^{E}(t+\Delta t)-a_{W} U_{\jmath}^{W}(t+\Delta t)-R_{\jmath}^{\min }(t+\Delta t) \Delta x=0
$$

where $\Delta t$ is the time step and $(t)$ refers to the values at the present time level. As discussed above, the equations are written in terms of the concentrations of the primary species in the code GIMRT (see Equation 32) and are expressed in terms of the total concentrations here in order to be concise.

\subsection{Transport Algorithm in Operator Splitting Approach}

\subsubsection{Advection}

When solutions require accurate resolution of concentration fronts in advection-dominant problems, highresolution shock-capturing techniques are often employed. These solution techniques are typically based on explicit numerical schemes that are incompatible with a globally implicit solution of coupled transport and reactions (e.g., GIMRT). Decoupling (i.e., time splitting) the transport algorithm from the geochemical reaction algorithm relaxes the requirement for an implicit transport method, allowing the use of high-resolution shock-capturing techniques based on explicit numerical schemes. The principal tradeoff for the additional accuracy is a strict limit on the ratio of time-step to grid spacing, a limit that is inherent in explicit transport methods. Typically, this limit becomes most restrictive under steady-state or near steady-state conditions. (Note that steady-state does not mean static processes. In physical systems, steady-state occurs when dynamic processes are balanced or fully compensating.)

In OS3D, an explicit, third-order, total variation diminishing (TVD) numerical scheme (Gupta et al., 1991) is used to model non-dispersive advective transport. The technique is based on calculating an estimated concentration at the face of a grid cell that is dependent on the flow direction. In the case of flow in the positive $\mathrm{x}$-direction (i.e., $u>0$, the estimated concentration would be:

$$
C_{i+1 / 2}^{n+1 / 2}=C_{i}^{n}+\beta(r)\left(\frac{C_{i+1}^{n}-C_{i}^{n}}{2}\right)
$$

where

$$
\beta(r)=\operatorname{Max}\left(0, \operatorname{Min}\left[2,2 r, \frac{2+r}{3}\right]\right)
$$

and

$$
r=\left[\frac{C_{i}^{n}-C_{i-1}^{n}}{\delta x_{i}+\delta x_{i-1}}\right]\left[\frac{C_{i+1}^{n}-C_{i}^{n}}{\delta x_{i}+\delta x_{i+1}}\right]^{-1} .
$$

Similar calculations are performed for negative velocities and the method is generalized for multiple dimensions. The technique is third-order accurate in smooth regions, oscillation-free along concentration fronts, and does not exhibit overcompression of fronts in the presence of physical dispersion. 


\subsubsection{Diffusion and Dispersion}

After the advection calculation, OS3D proceeds to solve for diffusion and dispersion. OS3D assumes that the principal direction of flow is aligned with the grid (i.e., $\mathrm{D}$ is a diagonal tensor). A centered numerical scheme, second-order accurate in space and time, is applied to the diffusion equation. The diffusion/dispersion equations are solved with the WATSOLV sparse matrix solver of VanderKwaak et al. (1995).

The user should understand that parameterizations of hydrodynamic dispersion attempt to account for advection resulting from variations in the velocity field that cannot be resolved by the numerical grid. In this standard representation based on Ficks law, dispersion is driven by the gradient of concentration and parameterized by a linear proportionality constant (i.e., the diffusion coefficient). This is a gross simplification of a complex and poorly understood phenomenon. As such, the formulation neglects many features that have been observed in real systems. Principally, the user should be aware that unlike molecular diffusion, dispersion is length and time scale- dependent.because of the inhomogeneity of the physical media. As plumes start to spread, they are exposed to larger and larger scales of spatially variable material properties. Consequently, it is impossible to define dispersion parameters that are effective for all time. Moreover, the magnitude of the dispersion parameter is dependent upon the grid resolution: larger for large grid cells and smaller for small grid cells.

\subsection{Solving the Equations with Newton's Method}

As an example, consider a problem involving one-dimensional multicomponent reactive transport. Equation 55 for any component $J$ at the grid point $P$ is a nonlinear function of the concentrations of the primary species at the the $P$ grid cell in the case of OS3D and of the $P, E$, and $W$ nodal points in the case of GIMRT. An iterative scheme, therefore, is required to solve the set of nonlinear equations in either case. Both codes solve the nonlinear equations with Newton's method which makes use of a Taylor series expansion to linearize the equations. Using Newton's method, the linearized set of equations for the 1D global implicit case become

$$
\sum_{\jmath^{\prime}=1}^{N_{c}} \frac{\partial f_{\jmath}^{P}}{\partial C_{\jmath^{\prime}}^{P}} \delta C_{\jmath^{\prime}}^{P}+\sum_{\jmath^{\prime}=1}^{N_{c}} \frac{\partial f_{\jmath}^{P}}{\partial C_{\jmath^{\prime}}^{E}} \delta C_{\jmath^{\prime}}^{E}+\sum_{\jmath^{\prime}=1}^{N_{c}} \frac{\partial f_{\jmath}^{P}}{\partial C_{\jmath^{\prime}}^{W}} \delta C_{\jmath^{\prime}}^{W}=-f_{\jmath}^{P},
$$

where $f_{j}$ is the discretized differential equation for the total concentration of each of the primary species. In a $2 \mathrm{D}$ problem, one may have dependencies, of course, on other neighboring grid cells in the $\mathrm{Y}$ direction (i.e., north and south of the grid cell of interest). In the case of the operator splitting or sequential iteration approach employed in OS3D, the Newton step only involves homogeneous and heterogeneous reactions within the grid cell itself since the transport is handled separately (i.e., there are no nonlinear terms involving spatial derivatives). The Jacobian matrix then takes the form

$$
\sum_{\jmath^{\prime}=1}^{N_{\mathrm{c}}} \frac{\partial f_{\jmath}^{P}}{\partial C_{\jmath^{\prime}}^{P}} \delta C_{\jmath^{\prime}}^{P}=-f_{\jmath}^{P}
$$

The individual terms, $\partial f_{j} / \partial C_{\jmath^{\prime}}$, make up the elements of the Jacobian submatrices. From Equation 55 , it follows that the Jacobian submatrices can be written further as

$$
\frac{\partial f_{\jmath}^{P}}{\partial C_{\jmath^{\prime}}^{P}}=\frac{\partial U_{j}^{P}}{\partial C_{\jmath^{\prime}}^{P}}\left(\frac{\Delta x}{\Delta t}+a^{P}\right)-\frac{\partial R_{j}^{\min }}{\partial C_{\jmath^{\prime}}^{P}} \Delta x
$$




$$
\frac{\partial f_{\jmath}^{P}}{\partial C_{\jmath^{\prime}}^{E}}=-a^{E} \frac{\partial U_{\jmath}^{E}}{\partial C_{\jmath^{\prime}}^{E}}
$$

and

$$
\frac{\partial f_{j}^{P}}{\partial C_{\jmath^{\prime}}^{W}}=-a^{W} \frac{\partial U_{j}^{W}}{\partial C_{\jmath^{\prime}}^{W}}
$$

From Equation 12 and Equation 9, the partial derivatives of the total concentrations with respect to the primary species concentrations are (Lichtner, 1992)

$$
\frac{\partial U_{\jmath}}{\partial C_{\jmath^{\prime}}}=\left\{\begin{array}{cc}
1+\sum_{\imath=1}^{N_{x}} \nu_{\jmath^{\prime} \imath} C_{\imath}\left(\frac{\nu_{\jmath^{\prime}}}{C_{J^{\prime}}}+\frac{\partial I}{\partial C_{\jmath^{\prime}}}\left[\sum_{k} \nu_{k \imath} \frac{d \ln \gamma_{k}}{d I}-\frac{d \ln \gamma_{2}}{d I}\right]\right) & j=j^{\prime} \\
\sum_{\imath=1}^{N_{x}} \nu_{\jmath^{\prime} \imath} C_{\imath}\left(\frac{\nu_{\jmath^{\prime}}}{C_{\jmath^{\prime}}}+\frac{\partial I}{\partial C_{\jmath^{\prime}}}\left[\sum_{k} \nu_{k \imath} \frac{d \ln \gamma_{k}}{d I}-\frac{d \ln \gamma_{2}}{d I}\right]\right) & j \neq j^{\prime}
\end{array}\right\}
$$

where $I$ is the ionic strength, the subscript $\imath$ refers to the secondary species, and $k$ is a (second) dummy subscript referring to the primary species. The partial derivatives of the reactions rates are given by

$$
\frac{\partial R^{\min }}{\partial C_{\jmath^{\prime}}}=-\sum_{m=1}^{N_{m}} \nu_{\jmath^{\prime} m} A_{m} k_{m} \frac{Q_{m}}{K_{m}}\left(\frac{\nu_{\jmath^{\prime} m}}{C_{\jmath^{\prime}}}+\frac{\partial I}{\partial C_{\jmath^{\prime}}} \sum_{k} \nu_{k m} \frac{d \ln \gamma_{k}}{d I}\right),
$$

where any dependence of the rate law on the activities of species far from equilibrium has been neglected and only first order reactions are considered $(\mathcal{M}$ and $n=1)$. For the case where the activities of various species (e.g., $\mathrm{H}^{+}$) affect the far from equilibrium dissolution rate, additional terms appear in the partial derivatives (not given here). The partial derivatives of the ionic strength, $I$, with respect to the primary species can be written as (Lichtner, 1992)

$$
\frac{\partial I}{\partial C_{\jmath^{\prime}}}=\frac{z_{\jmath^{\prime}}^{2} / 2+\sum_{\imath} \nu_{J^{\prime}} z_{\imath}^{2} C_{\imath} /\left(2 C_{\jmath^{\prime}}\right)}{1-1 / 2 \sum_{\imath} z_{\imath}^{2}\left[\sum_{k} \nu_{k \imath} d \ln \gamma_{k} / d I-d \ln \gamma_{2} / d I\right]}
$$

and the derivatives of the activity coefficients with respect to the ionic strength are

$$
\frac{d \log \gamma_{k}}{d I}=-\frac{A z_{k}^{2}}{2 I^{1 / 2}\left(\left(1+B \stackrel{\circ}{a}_{k} I^{1 / 2}\right)^{2}\right.}+\dot{b}
$$

If we define $f_{j}$ as making up the elements of the vector $\mathbf{b}$ when taken over all of the grid points in the system and the $\partial f_{j} / \partial C_{j^{\prime}}$ as forming the elements of the global Jacobian matrix $\mathbf{A}$, then Equation 59 can be written in the familiar form

$$
\mathrm{A} \cdot \delta \mathrm{C}=\mathrm{b} .
$$

Once the $\delta C_{y^{\prime}}$ are computed, they are used to update the concentrations of the primary species

$$
C_{\jmath^{\prime}}^{\text {new }}=C_{\jmath^{\prime}}^{\text {old }}+\delta C_{\jmath^{\prime}} \quad\left(\jmath^{\prime}=1, \ldots, N_{c} \times M\right)
$$

This completes a single Newton iteration. The procedure is repeated until convergence of the function residuals (the $f_{j}$ 's) are reduced to a tolerance set in the code, i.e.,

$$
f_{\jmath} \approx 0
$$

Without basis switching, it may be necessary to use the logarithms of the concentrations since the concentration of a basis species (especially in the case of redox) may drop to a very small value. If the logarithms of the concentrations are used, it is still possible to solve such problems without basis switching, although 
in redox systems the code sometimes reduces the time steps many times in order to obtain convergence. In both OS3D and GIMRT, the logarithms of the concentrations are solved for if no basis switching is used and the linear concentrations are used if full basis switching every Newton iteration is used. The user should normally begin by running the codes without basis switching, since that will be faster if there are no non-convergences of the Newton iteration. If the code is either not converging within the maximum number of Newton iterations, then full basis switching should be tried. The user may also want to compare the iteration count with and without basis switching, since the bottom line is the total CPU required to solve a particular problem.

\subsection{Structure of the Jacobian Matrix}

It is instructive to examine the structure of the Jacobian matrix which arises from the discretization of the coupled reaction-transport equations, since it has a bearing on the choice of a method to solve the set of linear equations given by Equation 32. The Jacobian matrices exhibit a block tridiagonal structure in a one-dimensional system. The blocks making up the global matrix $\mathrm{A}$ are $N_{c}$ by $N_{c}$ submatrices corresponding to the primary species at any grid point. That is, if we are writing the conservation equation for $\mathrm{SiO}_{2, a q}$ at a particular point in space, the equation $f_{\mathrm{SiO}_{2, a q}}$ will be differentiated with respect to all $N_{c}$ primary species (e.g., $\mathrm{SiO}_{2, a q}, \mathrm{Al}^{+++}, \mathrm{K}^{+}$, etc.). As an example, consider a one-dimensional system described by 4 primary species which is discretized using 4 grid points (this is for illustrative purposes only, since hopefully one would not attempt to solve differential equations using only 4 grid points). The matrix $\mathbf{A}$ can be represented compactly as a series of $4 \times 4$ submatrices, $\mathbf{A}_{\jmath \jmath^{\prime}}$, such that

$$
\mathbf{A}=\left[\begin{array}{cccc}
\mathbf{A}_{11} & \mathbf{A}_{12} & 0 & 0 \\
\mathbf{A}_{21} & \mathbf{A}_{22} & \mathbf{A}_{23} & 0 \\
0 & \mathbf{A}_{32} & \mathbf{A}_{33} & \mathbf{A}_{34} \\
0 & 0 & \mathbf{A}_{43} & \mathbf{A}_{44}
\end{array}\right]
$$

Those readers with some familiarity with numerical methods will recognize that this is the structure of any finite difference formulation of a partial differential equation (e.g., the temperature equation), except that in this case the entries are submatrices rather than single coefficients. If we reduced the system to a single chemical component, then the forms of the temperature and reaction-transport matrices would be identical. The submatrices need not be filled entirely with non-zero elements, but, in general, they will be dense in systems with either a large number of minerals or a great deal of complexation. The diagonal submatrices ( $\mathbf{A}_{11}, \mathbf{A}_{22}$ etc.) contain contributions from both the heterogeneous reaction term, $R_{j}^{\text {min }}$, and from the total soluble concentrations (the $U_{j}$ 's). The offdiagonal submatrices, in contrast, contain only contributions from the total soluble concentrations at the neighboring grid points. Note that the global matrix $A$ is sparse (i.e., the ratio of zero to non-zero entries in the matrix is large) when the number of grid points is large (e.g., greater than 100), even though the submatrices (reflecting the coupling between the various species due to reactions) may themselves be fairly dense.

Any method used to solve the set of equations described by Equation 59 which arise in the case of the global implicit method should take into account both the sparseness of the Jacobian matrix A. In the code GIMRT, the equations are solved now using the WATSOLV package (VanderKwaak et al., 1995). In the WATSOLV package, one may use either the GMRES algorithm or the CGSTAB algorithm developed by the authors. Users should consult the manual prepared by these authors (VanderKwaak et al., 1995) on the various input options which can be specified. At the present time, all of these input options are in data statements at the beginning of the driver routine gimrt. $f$. We have found, however, that for redox problems (and probably any similar problem in which there is a large range of concentrations) that the convergence criteria need to be set much smaller than indicated by VanderKwaak et al. (1995) in their manual. We found that the Newton iterations failed to converge when the tolerance was too loose and a 
level of preconditioning equal to 0 was used (see the WATSOLV manual). This could be remedied by either setting the level of preconditioning to 2 or 3 or by simply tightening up considerably the tolerance and using a level of 0 . This second option appears to give the best results.

\subsection{Convergence Criteria}

Convergence is obtained where all of the function residuals have been reduced below a tolerance set by the parameters atol and $r t o l$ in the driver routine gimrt. $f$ and in the file newton. $f$ in the case of the code OS3D. These parameters are located in data statements at the top of the named routines. The tolerance on function residuals is computed from

$$
\text { tolmax }=a t o l+r t o l\left(C_{\jmath}\right),
$$

where $C_{\jmath}$ refers to the primary species concentrations. If they are changed, the code should be recompiled. The functions residuals, when multiplied by the timestep delt in order to compare to the tolerances atol and rtol, have units of moles per $\mathrm{m}^{3}$ bulk volume. The default setting for the absolute tolerance is $10^{-9}$ mole $\mathrm{m}^{-3}$ which corresponds to $10^{-12}$ mole liter ${ }^{-1}$. Convergence will also be obtained where either the absolute value of the log concentration correction or the linear concentration correction drops below $10^{-15}$ (i.e., approaching the roundoff error of the computer).

\subsection{Updating Mineral Properties}

When convergence is achieved, the mineral volumes and surface areas (and porosity, if desired) are updated. The individual mineral volume fractions, $\phi_{m}$, are updated with the expression

$$
\phi_{m}(t+\Delta t)=\phi_{m}(t)+V_{m} r_{m}(t+\Delta t) \Delta t
$$

where $V_{m}$ is molar volume of the mineral and $r_{m}$ is its reaction rate. The user may either choose to leave the porosity constant (in which case its value is determined by the user in the input file), or the user may choose to update the porosity. Where updated, the porosity is computed from the

$$
\phi=1-\sum_{k=1}^{N_{m}} \phi_{m} .
$$

Note that in this formulation, both mineral volume fractions and reactive surfaces are assumed constant for any one time step. This decoupled, two step approach normally works well since the volume fractions of the minerals usually evolve much more slowly than the solute concentrations. However, in certain cases, the approach may lead to inaccurate solutions. The code has a check to make sure that the volume fractions of a dissolving phase does not go below zero, in which case a mass balance error would occur. Where the code indicates that this is occurring frequently, the time step should be reduced. In addition, one can specify a maximum rate of increase (using the parameter vpptmax described below) or decrease (using the parameter vdissmax described below) of the mineral volume fractions. The two step approach employed here may require smaller time steps when either the dissolving or precipitating phase has a high solubility or where very little of it is present. Where only small amounts of a mineral are present, than it is possible that dissolution of the entire amount present within a grid volume may occur in a single time step. This typically results in either inaccurate propagation of a mineral front or in oscillations in the mineral volume fraction profile. 
Initial reactive surface areas are specified along with initial mineral volume fractions by the user at startup. Reactive surface areas of minerals are updated differently at the present time depending on whether the minerals are primary or secondary. For primary minerals, the surface areas are given by

$$
A_{m}=A_{m, 0}\left\{\begin{array}{cl}
{\left[\left(\frac{\phi}{\phi_{0}}\right)\left(\frac{\phi_{m}}{\phi_{m, 0}}\right)\right]^{2 / 3}} & \text { dissolution } \\
{\left[\frac{\phi}{\phi_{0}}\right]^{2 / 3}} & \text { precipitation }
\end{array}\right\} \text {, }
$$

where $\phi_{m, 0}$ is the initial volume fraction of the mineral $m$ and $\phi_{0}$ is the initial porosity of the medium. This formulation ensures that as the volume fraction, $\phi_{m}$, of a mineral $\rightarrow 0$, its surface area also $\rightarrow 0$. In addition, for both dissolving and precipitating minerals the term $\left(\phi / \phi_{0}\right)^{2 / 3}$ requires that the surface area of a mineral in contact with fluid $\rightarrow 0$ when the porosity of the medium $\rightarrow 0$. For secondary minerals (i.e., those initially with a volume fraction $=0$ ), the reactive surface areas as a function of time are given by

$$
A_{m}=A_{m, 0}\left\{\begin{array}{cc}
{\left[\left(\frac{\phi}{\phi_{0}}\right) \phi_{m}\right]^{2 / 3}} & \text { dissolution } \\
{\left[\frac{\phi}{\phi_{0}}\right]^{2 / 3}} & \text { precipitation }
\end{array}\right\},
$$

which implies that if a secondary were to begin to dissolve, its surface area would equal its "initial" surface area, $A_{m, 0}$, when $V_{m}=1$ (if the porosity were constant).

\section{PROGRAM STRUCTURE}

\subsection{Global Implicit}

The basic structure of the global implicit approach (GIMRT) is summarized in Figure 2. After specifying the initial and boundary conditions, the code begins stepping through time. Within each time step, a series of Newton iterations is required because of the nonlinearity of the equations. Each Newton iteration consists of calculating the function residuals (the $f_{j}$ 's given by Equation 55 ) and the partial derivatives of these functions (the Jacobian elements) with respect to the unknown concentrations (Equation 59) and then assembling and solving the linear set of $N_{c} \times M$ algebraic equations in order to obtain the corrections to the component concentrations. If the basis switching option is turned on, then the code checks that the basis species chosen are those that form a linearly independent set and that are present in the greatest concentration.

If more concentrated species are present than those currently used for the primary species, then a basis switch is carried out at the appropriate grid cell (i.e., the reactions are rewritten in terms of a new set of primary species). Unlike the operator splitting approach in OS3D, the residual functions and the Jacobian matrix are computed over the entire spatial domain before solving a global system of linearized equations. Once convergence is achieved, the mineral volume fractions and mineral surface areas are updated. If the reaction-transport calculations are combined with a calculation of the flow and temperature field, these would normally be updated as well at this time. At the end of each time step, an algorithm which computes the second derivative of the solute concentrations with respect to time is used to determine whether the time step should be increased or decreased. This algorithm provides a way of minimizing the time truncation error (if that is an issue in a particular problem) and also of controlling the size of the time step so as to maintain numerical stability.

The program structure for the time splitting (operator splitting and sequential iteration) method is similar, differing primarily in the fact that the transport is carried out in advance of any calculations of the reactions 
(Figure 3). The reaction calculations are then calculated individually at each point in space. Since these reactions are not directly coupled via transport to the reactions in neighboring cells, the calculations can be carried out independently. The size of the system of equations to be solved at each grid point is then $\mathrm{N}_{c}$ by $\mathrm{N}_{c}$ and there is no need to assemble the global system of function residuals and the global Jacobian matrix over the entire spatial domain. The sequential iteration method differs in that $1 / 2$ of the reaction rate is included as a source term in advance of the "reaction module" and so iteration between transport and reaction is required to achieve convergence (Figure 3 ). Mineral volume fractions and the porosity are updated after all of the species have been reacted at all the space points, in a similar fashion to the global implicit.

\section{FEATURES OF THE CODE}

Before running the code, the user of OS3D and GIMRT needs to decide what physical process(es) he or she wants to model and design the simulation accordingly. This involves choosing the boundary conditions for the problem (e.g., no flux boundaries or fixed concentration boundaries) and setting up the initial grid and/or initial mineral zones. The user must decide how many grid points are needed to solve a particular problem. Typically one proceeds by carrying out the simulations on a relatively coarse grid (e.g., 30 to 50 grid points) and only refining the grid later when one has a general idea of the behavior of the system. The user also needs to decide what level of chemical complexity they wish to consider. This can involve choices in the number of independent components (primary species), the number of secondary species, and the number of reacting minerals. The discussion in the sections which follow is designed to make the basis for these decisions clearer. These sections are then followed by a step by step discussion of the input file threedin which is needed to run OS3D and GIMRT.

\subsection{Units}

Although there are some advantages to allowing the input of data in any self-consistent set of units, many of the parameters used in GIMRT and OS3D are given commonly in certain units, so we have decided to require these units. All units for input parameters (e.g., velocities, reaction rates etc.) are given in the description of the input file threedin. All distances are in meters. The code assumes that the reaction rates for minerals are in units of mol m $\mathrm{m}^{-2} \mathrm{~s}^{-1}$ (these are converted in the code to mol $\mathrm{m}^{-2} \mathrm{yr}^{-1}$. Diffusion coefficients are in units of $\mathrm{m}^{-2} \mathrm{~s}^{-1}$. All other references to time (time for plot files, timesteps etc.) are in years. Velocities, for example, are in units of $\mathrm{m} \mathrm{yr}^{-1}$. Concentrations for aqueous species are in units of mol kg ${ }^{-1}$ water (i.e., molality). The partial pressure of gases are specified in bars.

\subsection{Boundary Conditions}

At the present time there are three possible boundary conditions which can be used in GIMRT and OS3D. The most straightforward way to describe the possible boundary conditions in the codes is to actually begin with the third type of boundary (also sometimes called a Danckwerts boundary conditon). This is a boundary condition which requires continuity of flux across the boundary. Both codes assume that the flux at these boundaries is purely advective (i.e., no diffusion or dispersion occurs upstream). Where the flow is into the system, the flux at the first interior nodes downstream is assumed to be just $u C_{\text {ext }}$, the same as the advective flux at the upstream, exterior node. In this special case where the dispersive and diffusive flux are neglected, the third type of boundary condition is the same as a Dirichlet or fixed 


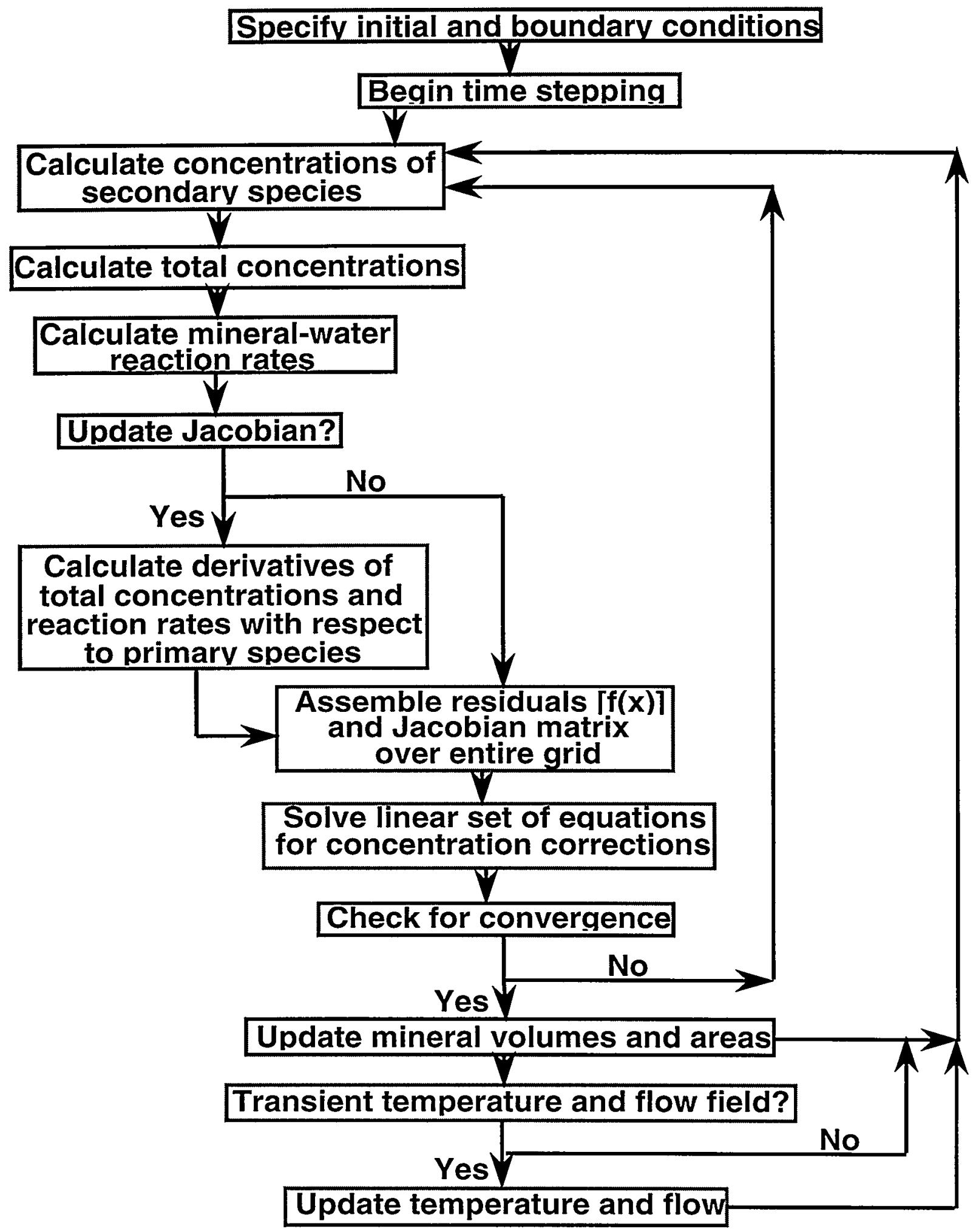

Figure 2: Program structure for GIMRT which uses a global implicit or onestep method used to solve the multi-component reaction-transport equation. 


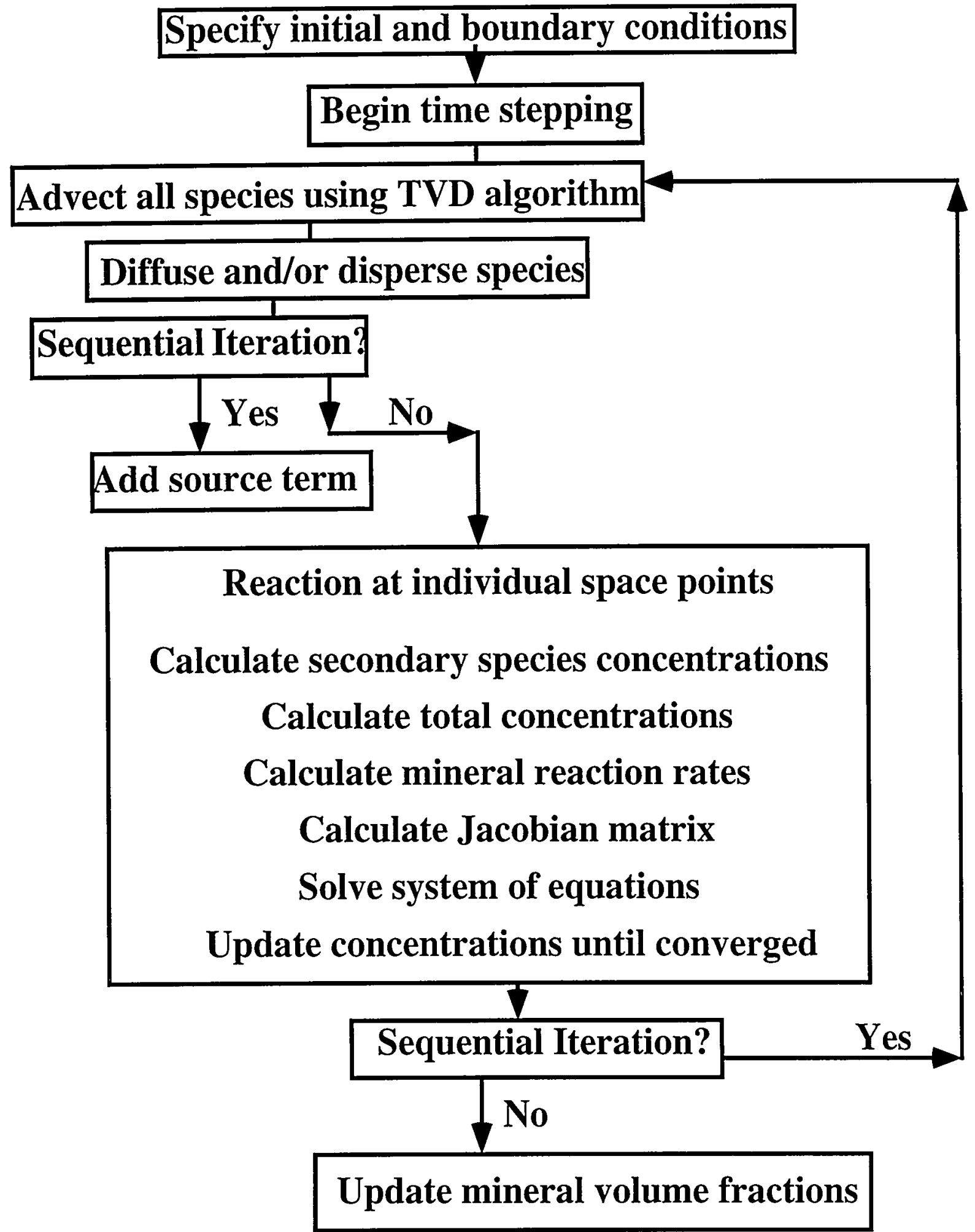

Figure 3: Program structure for OS3D which uses time splitting of the transport and reaction step or a sequential iteration between transport and reaction.

concentration boundary condition. At the boundary nodes where the flow passes out of the domain the flux is also assumed to be purely advective, so that it passes out of the system with the downstream, exterior node having no effect on the system. Note that when the flow across a boundary is non-zero, we always assume that the dispersive and diffusive fluxes are equal to zero. 
The second type of boundary condition is the no-flux condition which is applied when there is no flow across the boundary. In this case, both the advective flux and the dispersive/diffusive fluxes are equal to zero.

The last type (often called the first type or a Dirichlet boundary condition) is normally applied when the advective flux is equal to zero but there is a dispersive or diffusive flux across the boundary (if the advective flux is non-zero, then the code automatically assumes a zero dispersive/diffusive flux). This condition is many cases is not physically reasonable, since it assumes that the diffusive and/or dispersive flux through the boundary does not affect the concentration of the exterior node (i.e., it is fixed). This boundary condition would normally be applied where the physical processes exterior to the domain are such that the diffusive/dispersive flux through the boundary is not enough to alter the concentrations there. One example might be a boundary along a fracture within which the flow is so rapid that the diffusive flux through the fracture wall has a negligible effect (e.g., Steefel and Lichtner, 1994). Another example might be a boundary corresponding to an air-water interface where a gas like $\mathrm{O}_{2}$ or $\mathrm{CO}_{2}$ is present in such large abundance that it effectively buffers the concentrations in the aqueous phase. Another example which is often mentioned in this regard is the case where mineral equilibria fixes the concentrations. This is dangerous, however, since the system is only completely determined in the special case where the phase equilibria results in an invariant point, i.e., no thermodynamic degrees of freedom exist. This is extremely rare in open systems, despite the frequent invocation of this condition in metamorphic petrology. Normally, since the concentrations will not be uniquely determined, a rigorous treatment would require that a speciation calculation with equilibrium constraints be carried out to actually determine what the concentrations at the exterior node should be. This, however, is not implemented at the present time.

OS3D and GIMRT differ slightly in their application of a Dirichlet boundary condition where the advective fiux across the boundary is zero. In the case of GIMRT, the user may specify that an exterior node be a fixed concentration node and that it affect the interior node (i.e., there will be a non-zero diffusive and/or dispersive flux through the boundary). In OS3D, however, the code always assumes the dispersive/diffusive flux across the boundary is zero (due to the way the transport algorithm is formulated). If one wants to use a Dirichlet condition in the case of OS3D, one does so by fixing the concentrations at the first node or nodes interior to the boundary. This will have the same effect as allowing an exterior node influence the interior domain, except that one can think of the boundary as having been moved inward by one grid cell. The procedure for doing this is described in the section below explaining the input file threedin.

To summarize the boundary condition options in OS3D and GIMRT, a non-zero flow across the boundary means that the code will use the upstream, exterior boundary concentrations to give a purely advective flux at the boundary and the code will allow a fluid packet to pass out of the system with no influence from a downstream, exterior nodes. Exterior nodes across a boundary through which there is no flow will not influence the interior domain, unless the Dirichlet boundary condition option is used (usage described below). The default is therefore no diffusive/dispersive flux boundaries which is only overridden where the Dirichlet option is invoked.

\subsection{Choice of Primary and Secondary Species}

After setting up the problem physically, one needs to decide on the chemistry to be included. The method for specifying primary and secondary species is essentially that devised by Peter Lichtner for the code MPATH. The codes OS3D and GIMRT require that one specify an initial choice of primary species which then determine the number of independent chemical components in the system. This choice of primary species is not unique, however, as discussed in the section 2.2. By making use of the basis switching 
option, it is possible to allow the code to dynamically choose the set of primary species to be used in writing the reactions at each grid point (see discussion below). Unlike EQ3/EQ6 (Wolery et al., 1990), for instance, the user must choose which secondary species are to be included in the simulation. This can be an advantage for multicomponent reaction-transport modeling since one would like the option of carrying out chemically simplified simulations. However, the user must take care that important species are not neglected. Future versions of the code will have the capability of reading all of the potentially relevant species from the database.

The only cautionary note here is that one must include either among the primary or secondary species a species which is used in writing the reaction in the EQ3/EQ6 data base. Note that this database is provided with the code in a reformatted form. For example, if one wants to include aluminum in the simulation, then the species $\mathrm{Al}^{+3}$ must appear among either the primary or secondary species since that species is used as the primary or basis species in the EQ3/EQ6 data base. In the same way, one must include $C O_{2(g)}$ among the list of gases if carbon is to be present in the system. If this is not done, the code will not be able to find the reaction when searching the data base. One should not, however, include any secondary species which cannot be written completely in terms of the basis species specified in the input file. The simplest way to grasp these features is to check the database to see which species are used to write the reactions involving a species of interest.

The species $\mathrm{H}_{2} \mathrm{O}$ presents a special case since in many cases it is present in such abundance that its activity can be taken as unity. If one wants the activity of $\mathrm{H}_{2} \mathrm{O}$ to be 1 , then it should be left out of the list of primary and secondary species. The code will still balance the reactions properly as if $\mathrm{H}_{2} \mathrm{O}$ were present. If, on the other hand, one wants to carry out a mass balance on $\mathrm{H}_{2} \mathrm{O}$, then it should be included in the list of primary species and it will be treated like any other species, except for the treatment of its activity coefficient. OS3D and GIMRT assume that the activity of $\mathrm{H}_{2} \mathrm{O}$ is given by its mole fraction in the solution.

\subsection{Redox Reactions}

There are many possibilities for choices of primary and secondary species to represent redox reactions. Both OS3D and GIMRT write all redox reactions as whole cell reactions, i.e., electrons are not used as either primary or secondary species. It is easy to show that the electrons are completely unnecessary if the solutions are electrically neutral, and the inclusion of electrons requires that one include a species the mass of which should everywhere be zero. With the whole cell reactions, no special provision for redox species and reactions is necessary, (in contrast to statements by Yeh and Tripathi (1989)) although the inclusion of redox reactions implies that we have included an additional mass balance equation representing the conservation of exchangeable charge.

Since $O_{2(g)}$ is used as the basis species in redox reactions in the EQ3/EQ6 data base, it must be included in the list of gases in the input file if redox reactions are to be considered. A gas, however, cannot be used as a primary species in OS3D and GIMRT since gases are not included in the mass balances. One could use $\mathrm{O}_{2(a q)}$, however, or any number of other species which form a redox couple, for example, $\mathrm{Fe}^{+2}$ and $\mathrm{Fe}^{+3}$, or $\mathrm{HS}^{-}$and $\mathrm{SO}_{4}^{-2}$. When using the basis switching option, the initial choice of primary species to represent a redox couple may not be important since the code will automatically write the reactions at any particular grid point in terms of the dominant redox couple, i.e., that which is present in the largest concentration (see section on Basis Switching below). This may be particularly useful for redox problems which can show convergence problems (this approach works best where basis switching is carried out every Newton iteration). The user may choose not to use basis switching at all, to use it at the beginning of every timestep, or to use it every Newton iteration. The same proviso, however, applies about including 
the species which are used as basis species in the EQ3/EQ6 data base.

\subsection{Acid-Base Reactions}

Acid-base reactions require no special treatment other than in the initialization process where one normally fixes or calculates $\mathrm{pH}$ rather than an $\mathrm{H}^{+}$concentration. Once a $\mathrm{pH}$ has been either fixed or calculated in the initialization procedure, however, $\mathrm{H}^{+}$can be treated like any other component. Again, no special considerations are needed in contrast to the statements by Yeh and Tripathi (1989).

\subsection{Basis Switching}

As discussed above, both OS3D and GIMRT can rewrite the reactions in terms of the most concentrated set of linearly independent species. This is not absolutely necessary if one is solving for the logarithms of the concentrations, but basis switching, when carried out every Newton iteration, does appear to reduce the number of times the Newton fails to converge, particularly in the case of redox problems. If full basis switching is used (every Newton iteration), it should not be necessary to solve for the logarithms of the concentrations and one can make use of standard relaxation techniques to reduce the size of the Newton step when the linear concentrations are employed. The code carries out basis switching by first sorting the species concentrations in order of abundance and then systematically attempting to construct a linearly independent set from those concentrations (see the subroutine switch.f). The recommended procedure is to try to run the problem without basis switching, since that will be the fastest method if the code converges properly. If numerous non-convergences occur, however, we recommend trying basis switching. The various input parameters required to turn the option on and off and to set the maximum allowable concentration correction limits are described in detail in the section on the input file threedin.

\subsection{Activity Coefficient Model}

At this time, the user may choose to run the code either with unit activity coefficients or with an extended Debye-Hückel formulation to calculate activity coefficients for the ionic species. The Debye-Hückel formulation is given by

$$
\log \gamma_{i}=-\frac{A Z_{i}^{2}(I)^{1 / 2}}{1+B \stackrel{\circ}{a}_{i}(I)^{1 / 2}}+\dot{b} I
$$

The coefficients for the temperature dependence of the parameters are taken from the EQ3 data base.

\subsection{Initialization Procedure}

The codes GIMRT and OS3D initialize the solute concentrations at individual grid points over the domain by carrying out a distribution of species using a number of options. The initial concentrations of the primary species may be determined by

- carrying out a mass balance on a total concentration (e.g., aluminum),

- carrying out a charge balance on a species (e.g., $\mathrm{Cl}^{-}$), 
- a mineral equilibrium constraint (e.g., $H^{+}$required to be in equilibrium with calcite),

- fixing the activity or partial pressure of a gas (e.g., $\mathrm{CO}_{2(g)}$ ),

- fixing the activity (e.g., $\mathrm{pH}$ ) of a species,

- fixing the concentration of a species, and

- fixing the number of moles surface hydroxyl sites per mole mineral and the intrinsic surface area of the mineral (i.e., $\mathrm{m}^{2} / \mathrm{g}$ ).

This last option is available in OS3D and GIMRT and it allows one to specify the concentration of surface complexes. These are specified by giving a site density (mole per $\mathrm{m}^{2}$ mineral) and a surface area of mineral per gram mineral. The total concentration of surface hydroxyls developed on the mineral (converted to $\mathrm{mol} / \mathrm{kg}$ solvent) is then computed from this information and the abundance of the mineral specified in the startup file. If a mineral is initially is not present but one would like to consider adsorption on this mineral, then the total concentration of surface hydroxyls on this mineral is set to a small number $\left(10^{-10}\right.$ $\mathrm{mol} / \mathrm{kg}$ ) and its concentration only builds up once the secondary mineral precipitates.

How each of the above initialization constraints is applied is described in the section on the input file threedin below.

\subsection{Temperature Gradients}

Since GIMRT and OS3D are based on finite difference methods, they have no problem handling temperatures which are non-constant either in time or in space, although this option has been implemented only in GIMRT due to time constraints on the preparation of the software. If either a non-default thermodynamic data base or the master25. data data base is not used at startup, then the code defaults to the mastertemp. data file where equilibrium constants are given at temperatures of $0^{\circ} \mathrm{C}, 25^{\circ} \mathrm{C}$, $60^{\circ} \mathrm{C}, 100^{\circ} \mathrm{C}, 150^{\circ} \mathrm{C}, 200^{\circ} \mathrm{C}, 250^{\circ} \mathrm{C}$, and $300^{\circ} \mathrm{C}$ at pressures corresponding to the water saturation curve. The code then fits a polynomial to the thermodynamic data so that equilibrium constants can be generated at any temperature between $0^{\circ} \mathrm{C}$ and $300^{\circ} \mathrm{C}$ along the water saturation curve. Simulations are possible at other pressures and temperatures, but the user must provide his or her own data base in the same format as master 25 . data or mastertemp. data. In addition, the algorithm for fluid density must be modified as well. Presently the fluid density is calculated as a function of temperature from a polynomial fit to the data in Helgeson and Kirkham (1974). Fluid viscosity is calculated from the data presented by Bruges and others (1966).

A temperature dependence is also included in the calculation of the reaction rate constants (Equation 29), the diffusion coefficients, and the fluid densities. Reaction rate constants at temperature are obtained normally by extrapolating the rate constants for $25^{\circ} \mathrm{C}$ given in the input file threedin. If one would rather input directly the desired rate constant at temperature, than the simplest procedure (short of changing the source code) is to input the rate as if it were the $25^{\circ} \mathrm{C}$ data while changing the activation energy to 0 $\mathrm{kcal} / \mathrm{mole}$. There is also an option for directly inputting the diffusion coefficient at temperature.

There are two options for temperature presently implemented in GIMRT. The first is simply to run the simulation isothermally, either at $25^{\circ} \mathrm{C}$ or at temperature. The second is to specify a linear temperature gradient which operates only in the $\mathrm{X}$ direction. Many other ways of handling temperature are possible, including allowing for a transient temperature field, but this must be provided by the user/programmer at the present time. This can be done with a simple read of temperatures, making sure that the dimensions 
of the temperature field match those specified in the code. The positions in the code where a user should place a routine for calculating or inputting a temperature are marked in the routine gimrt. f.

\subsection{Updating Porosity and the Transport Coefficients}

A user can choose at runtime whether to include a feedback between the changes in the volume fractions of the various minerals in the system and the diffusion coefficient. In the present version of the code, there is no provision for a feedback between porosity and permeability. This is planned as a feature of the next release. To do so requires that one solve the continuity equation (the equation for the conservation of fluid mass) in addition to the reaction-transport equation (Steefel and Lasaga, 1990; Steefel and Lasaga, 1994). Local changes in the porosity result normally in local changes in the permeability, thus causing a change in the distribution of the hydraulic head (or fluid pressure). The continuity equation should be solved in a one-dimensional system with constant head boundary conditions, and not constant flux conditions as was done in Berner (1980). The fluid velocity becomes infinite as the porosity $\rightarrow 0$ when the constant flux conditions are used.

If the user decides to update porosity during the course of the simulation, then the magnitude of the diffusion coefficient in the porous medium, $D^{*}$, changes according to Archie's Law (Dullien, 1979)

$$
D^{*}=\frac{D_{0}}{F}=\phi^{m} D_{0}
$$

where $m$ is again the "cementation exponent" in Archie's Law. This formulation ensures that $D^{*} \rightarrow 0$ as $\phi \rightarrow 0$. It should also ensure that the porosity will not go negative if the chemical reactions are driven by mass transport since the porous medium locally becomes a closed system.

\section{INSTALLATION}

The codes OS3D and GIMRT are designed at the present time to be installed and run on UNIX systems. The code has been tested to date on Sun, Digital (ULTRIX), Silicon Graphics, and IBM RS-6000 series UNIX-based workstations. A number of suggestions follow which are designed to make it easier to customize the installation.

The directories GIMRT and OS3D contain the subdirectories

- src, where the source files, Makefile, and include files are located,

- database, where the default data bases, master25. data and mastertemp data are located,

- doc, where the documentation in the form of a PostScript file is located.

The user needs to modify the source code so that the correct address of the GIMRT or OS3D directory tree can be found. The address of the data bases is specified in the file database. $f$ in the lines

c open appropriate data base file

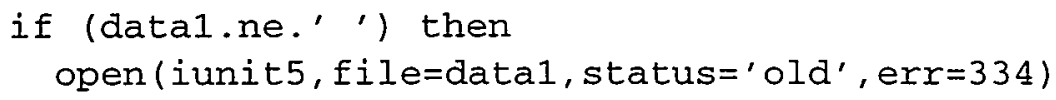




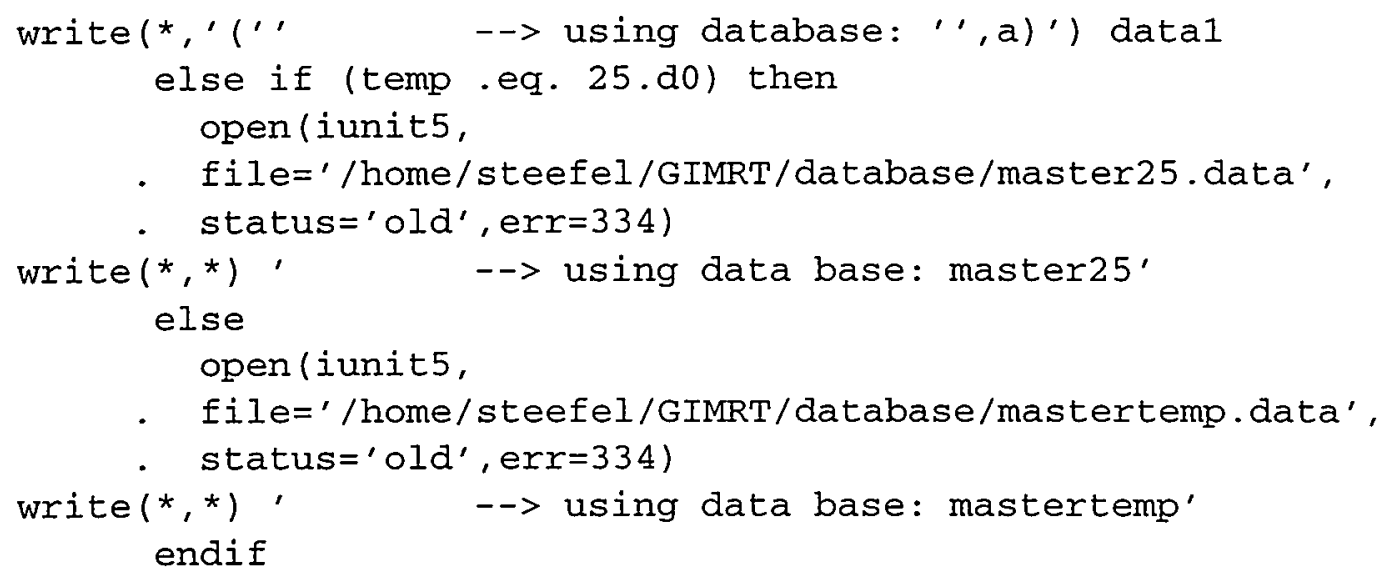

One of the options above involves a non-default data base or location (specified by data1 .ne.' ') which is discussed in the section on input files below.

The source code comes with Makefiles Makegimrt and Makeos3d which should be modified slightly depending on the particular operating system to be used. Copy the file Makegimrt or Makeos $3 \mathrm{~d}$ over to Makefile and uncomment the lines appropriate for the particular system on which the software is being installed (e.g., Sun, DEC, or IBM). These options at this time involve only slight differences in the compilation flags, and in the case of the IBM AIX operating system, the command to invoke the FORTRAN compiler.

The second file which may have to be modified before compilation is params. inc in the case of the GIMRT code or params_os. inc in the case of the OS3D code. This file contains the parameter statements which dimension the various arrays in the codes. The codes are set up so that at run-time the dimensions of the arrays are checked to make sure they are sufficiently large. If the user needs to increase the dimension of the arrays or wants to decrease them so that they are just large enough, the following parameters can be changed

- mcomp, 1 plus the number of independent chemical components in the system (specified in threedin),

- $m r c t, 1$ plus the number of reacting minerals in the system (specified in threedin),

- mspec, 1 plus the number of secondary species in the system (specified in threedin),

- mgas 1 plus the number of gases in the system (specified in threedin), and

- $m x, m y, m z$, the number of grid points in the $\mathrm{X}, \mathrm{Y}$, and $\mathrm{Z}$ directions respectively used to solve the for the solute concentrations (specified in threedin).

If the file params. inc or params_os. inc are modified, then all of the subroutines which include this file are recompiled when Makefile is run again.

When the appropriate changes have been made, the source code can be compiled and an executable image can be created. This is done by giving the command make which runs the default file Makefile.

So as to avoid the necessity of creating multiple copies of the executable image, one normally creates a symbolic link to the user's bin directory. This allows the code to be run from any directory chosen by the user (assuming the input file threedin is located there). This can be done by moving to the user's bin directory with the command

$$
c d \sim / b i n
$$


where $\sim$ indicates the user's home directory, and then typing

$$
\text { ln }-s \sim / p a t h / \text { gimrt gimrt }
$$

followed by

$$
\text { rehash }
$$

to update the hash table so that the symbolic link is recognized. The designation / path refers to the location of the executable image (e.g., /GIMRT). The user should also make sure that the / bin directory is included in their search path (generally in the .cshrc or . login file). The search path is set with the command set path.

\section{RUNNING GIMRT and OS3D}

OS3D and GIMRT both read the user-provided input file threedin on startup in addition to a file containing the thermodynamic data base. The file threedin includes various control parameters and physical parameters which are necessary in order to run the code. The file also includes information on the initial and boundary conditions, the minerals and species to be included, and the rate data to be used in the simulation. The input is organized as a series of cards which include comment lines followed by the fields where the user inputs data. Comment lines begin all cards which are numbered. Input data begins on the second line after the last comment line (i.e., comments and input are separated by one space).

\subsection{Control Parameters}

Control and physical parameters for the simulation are specified in Cards 1 through 7 of the input file threedin. A typical example is given below.

* THIS IS THE INPUT data TO GIMRT AND OS3D*

$$
\text { title (up to } 80 \text { characters) }
$$

'Short Course Test Problem'

$$
\text { database ( } 1 \text { blank space for default) }
$$

$$
\text { tstep (max. delt), delt (initial delt), ttol, ijacob,bpeg }
$$
0.005
$1 . e-06$
0.01
0
$5 \cdot d-03$

jpor, constantpor (porosity), vdissmax, vpptmax

$$
0.1 \quad 0.01 \quad 0.001
$$

jtemp, tinit, tgrad 
$25.0 \quad 0.0$

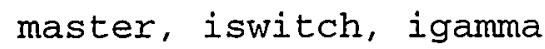

'h+' 2

nstop, (prtint(i), $i=1 \ldots$ nstop)

$13.020 .0 \quad 30.0 \quad 40.050 .0$

- Card (1) LTITLE Title of simulation (up to 80 characters) enclosed in single quotes.

- Card (2) DATA1 String (up to 100 characters) with location of a user-provided thermodynamic data base. If no location is provided (i.e, the code reads ' $'$ ), then the code defaults to either master 25 . data if the input temperature (given below) is $25^{\circ} \mathrm{C}$ or to mastertemp. data if another temperature is given.

- Card (3) TSTEP, DELT, TTOL, IJACOB, BPEG

TSTEP: maximum time step in years allowed.

DELT: initial time step in years.

TTOL: parameter controlling the tolerance on increasing the size of the time step. Making ttol smaller decreases the rate at which the time step is increased, for the purpose of controlling either the time truncation error or for numerical stability.

IJACOB: flag controlling update of Jacobian

0 - update Jacobian every Newton iteration

1 - when delt $=1 / 2$ tstep, update Jacobian every 3 Newton iterations and when delt $=$ tstep, update Jacobian every 6 iterations.

BPEG: maximum change in molality in any one Newton iteration. Reducing bpeg increases the under-relaxation of the Newton step and may sometimes prevent nonconvergence, although the total number of iterations required may actually increase.

\section{- Card (4) JPOR, CONSTANTPOR, VDISSMAX, VPPTMAX}

JPOR: flag controlling update of porosity and flow

0 - no update of porosity.

1 - porosity is updated.

CONSTANTPOR: porosity of the medium for the case where the porosity is not updated (jpor $=0$ ). If jpor $=0$, the porosity is taken from constantpor rather than from the sum of the volume fractions of the minerals in the system. If jpor $=1$ (porosity updated), constantpor is ignored.

VDISSMAX: maximum rate of decrease of a mineral's volume fraction (units of $\mathrm{yr}^{-1}$ ). Can be used to limit the amount of mineral dissolution which can occur within a single time step. Useful where either the dissolving phase is very soluble or where very small amounts are present. Where very small amounts of a mineral are present, inaccurate mineral front propagation may occur where most or all of the mineral present within a grid volume dissolves in a single time step. 
VPPTMAX: maximum rate of increase of a mineral's volume fraction (units of $\mathrm{yr}^{-1}$ ).

- Card (5) JTEMP, TINIT, TGRAD

JTEMP: flag controlling temperature option.

0 - isothermal.

1 - linear temperature gradient operating only in $\mathrm{X}$ direction.

TINIT: temperature in ${ }^{\circ} \mathrm{C}$ at grid point 1 .

TGRAD: temperature gradient in ${ }^{\circ} \mathrm{C}^{-1}$. A positive gradient causes the temperature to increase from grid point 1 , a negative gradient causes it to decrease.

- Card (6) MASTER, ISWITCH, IGAMMA

MASTER: string (up to 18 characters) enclosed in single quotes specifying master variable (used in controlling time step).

ISWITCH: flag controlling basis switching.

0 - no basis switching (solves for logarithms of concentrations).

1 - not allowed at this time.

2 - basis switching every Newton iteration. Improved method over earlier versions which involves solving for the linear concentrations and using relaxation to prevent overly large Newton steps or negative concentrations. Results in reduced number of cases where non-convergence occurs, especially in redox examples. Does require some extra CPU time, however.

IGAMMA: flag controlling method for computing activity coefficients.

0 - unit activity coefficients (except for $\mathrm{H} 2 \mathrm{O}$ ).

2 - extended Debye-Hückel without calculation of the derivatives of the activity coefficients with respect to the primary species.

- Card (7) NSTOP, PRTINT

NSTOP: number of graphics files to be printed out.

0 - no time stepping, distribution of species only.

$\geq 1$ - number of output files at prtint intervals.

PRTINT: times at which output should be written (at least nstop times should be listed.

\subsection{Specifying Primary and Secondary Species}

In the next part of threedin (Cards 8 through 9), the initial choice of primary and secondary species is specified. Each list is terminated with ' $*$ '. An example input would be:

PRIMARY SPECIES

$' \mathrm{cot} 2$ '

'tracer'

' $h+'$ 
'fe+3'

'al+3'

' $\operatorname{sio} 2(a q)$ '

'nat'

'cl-'

'hco3-'

'> feoh'

$1 * 1$

'oh-'

'aloh+2'

'al (oh) 2+'

'al (oh) 3 (aq)'

'al (oh) 4-'

' $\operatorname{co} 2$ (aq)'

' $\operatorname{co3}-2$ '

'h3sio4-'

'nacl (aq)'

'>feo-'

'>feoh $2+$ '

'> feoh-co'

, *

- Card (8) PRIMARY SPECIES

- $\mathrm{NAMC}(\mathrm{J})$ name of primary species to be included (enclosed in single quotes).

- ' $*$ ' marks end of primary species read.

In this example, $\mathrm{H}^{+}$is chosen as the primary species, implying that $\mathrm{OH}^{-}$has been chosen as a secondary species. A secondary species should be included only if it can be written completely in terms of the included primary or basis species. The exception to this is a species which is written in terms of $\mathrm{H}_{2} \mathrm{O}_{(a q)}$. The reaction will be correctly balanced whether or not one chooses to explicitly include $\mathrm{H}_{2} \mathrm{O}_{(a q)}$ among the primary species. The inclusion of $\mathrm{H}_{2} \mathrm{O}_{(a q)}$ only determines whether the code carries out a mass balance on $\mathrm{H}_{2} \mathrm{O}_{(a q)}$. Since the reactions involving carbonate in the $\mathrm{EQ} 3$ data base are written locally in terms of $\mathrm{CO}_{2(g)}$, it should be included in the list of gases towards the end of the input file. Similarly, the inclusion of redox reactions requires that $\mathrm{O}_{2(g)}$ be included in the list of gases since the EQ3 database writes many of the redox reactions using that species. Species beginning with a $>$ (as in $>f e o h$ ) are surface complexes which need to be added to the thermodynamic database.

\section{- Card (9) SECONDARY SPECIES}

- NAMCX(I) name (enclosed in single quotes) of the species to be included.

- ' $*$ marks end of secondary species read. 


\subsection{Minerals}

The reacting minerals to be included in the simulation are read in from card (10) with the user-provided rate data. An example of the format is given below.

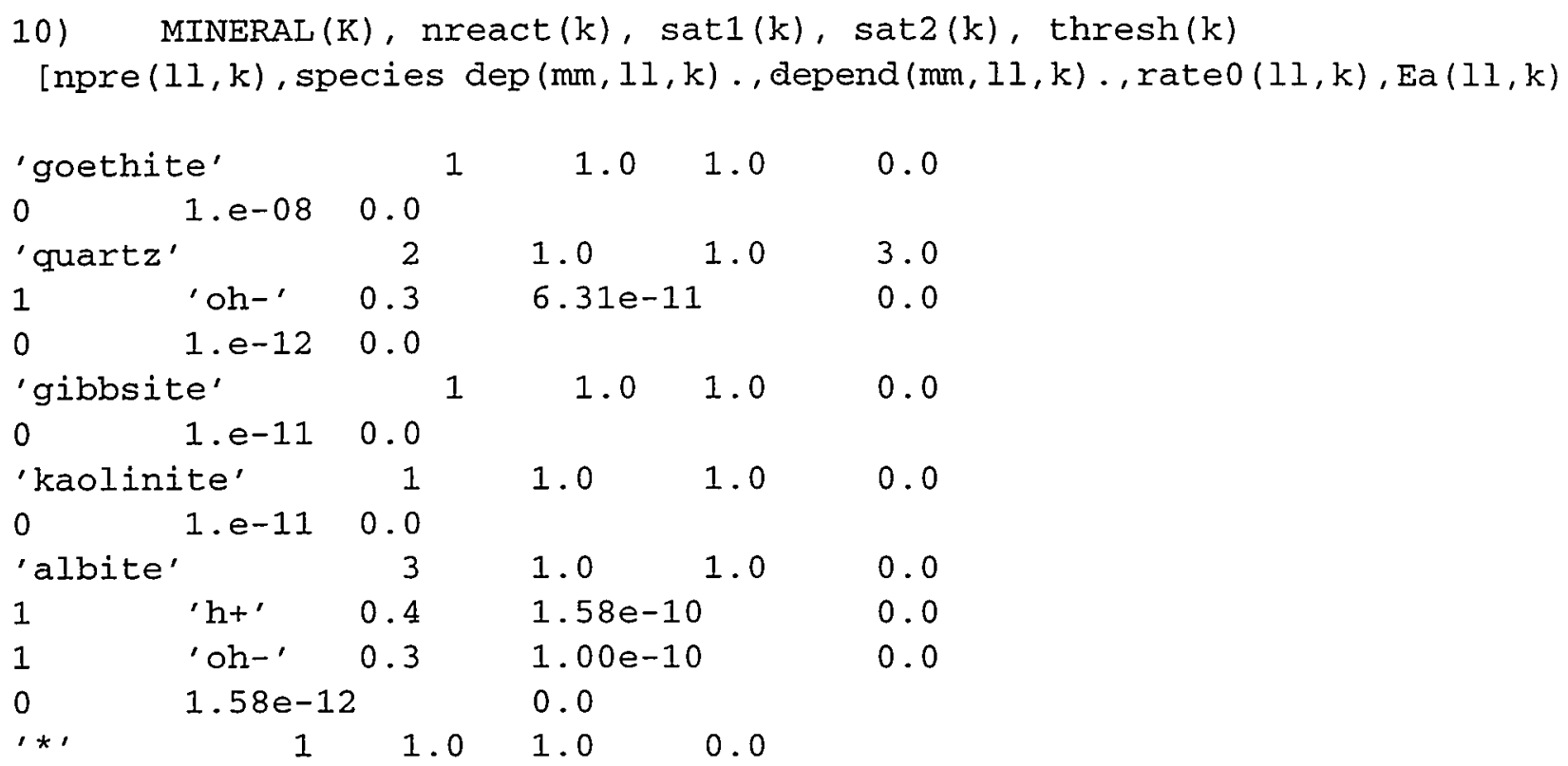

- Card (10) NAMK(K), NREACT(K), SAT1(K), SAT2(K), THRESH(K)

$\operatorname{NAMK}(\mathrm{K})$ : character string containing mineral name.

$\mathrm{NREACT}(\mathrm{K})$ : number of parallel reactions involving the mineral. The code will read nreact following lines.

SAT1(K): $\quad$ order of reaction rate law ( $n$ in Equation 32).

SAT2(K): $\quad$ order of reaction rate law ( $\mathcal{M}$ in Equation 32).

THRESH(K): supersaturation $(\log \mathrm{Q} / \mathrm{K})$ necessary for precipitation.

- NPRE(LL,K), DUMLABEL(MM) DEPEND(MM,LL,K), RATE0(LL,K), EA(LL,K)

NPRE(LL,K): number of species affecting the far from equilibrium dissolution rate. The code will then read npre character strings (dumlabel) and exponential dependences (depend).

DUMLABEL(MM): character string containing species name affecting far from equilibrium dissolution rate.

DEPEND(MM,LL,K): exponential dependence of the far from equilibrium rate on species given in preceding dumlabel.

RATEO(LL,K): far from equilibrium mineral dissolution rate constant at $25^{\circ} \mathrm{C}$ in units of moles $\mathrm{m}^{-2} \mathrm{~s}^{-1}$. Can be used as the rate constant at temperature if activation energy is set $=0$.

$\mathrm{EA}(\mathrm{LL}, \mathrm{K})$ : activation energy in units of $\mathrm{kcal}^{\mathrm{mole}} \mathrm{e}^{-1}$.

- ' $*$ ' end of mineral read. 
The user needs to be careful in inputting data in this section because of the range of options. After reading the mineral name and nreact, the number of parallel reactions involving the mineral, the code will read nreact following lines, each one corresponding to the parallel reaction. Each successive line corresponding to a separate reaction mechanism includes the number of species affecting the far from equilibrium dissolution reaction (see Equation 28). The code will then read on the same line npre character strings and exponential dependences. This is then followed (on the same line) by a read of the rate at 25 ${ }^{\circ} \mathrm{C}$ in units of moles $\mathrm{m}^{-2} \mathrm{~s}^{-1}$ and the activation energy. In this fashion, the user can specify multiple, parallel reactions (e.g., $\mathrm{pH}$ dependent and $\mathrm{pH}$ independent or ligand-promoted and proton-promoted reaction mechanisms) and within any one reaction mechanism, multiple dependences on.species far from equilibrium. All reactions, however, include a saturation state dependence which guarantees that the rate will go to zero when the mineral reaches equilibrium with the solution.

\subsection{Gases}

The format for inputting gases, which are not used in the mass balances, is the same as the format for secondary species and are read from Card 11.

- Card (11) NAMG(I) name (enclosed in single quotes) of the gas to be included.

- ' $*$ ' marks end of read.

\subsection{Discretization}

The next section of the input file threedin (Cards 12 through 14) includes user-provided information about the discretization to be used in the problem. All 3 dimensions must be specified even for 2D, 1D, or OD problems, since the code will expect to read three lines here. An example of a 1D problem using 32 grid cells in the $X$ direction with a grid spacing of 1.0 meter follows. Note that since the number of zones in the $\mathrm{Y}$ and $\mathrm{Z}$ directions are set to 0 , the information about the number of grid cells in the $\mathrm{Y}$ and $\mathrm{Z}$ directions is ignored. This problem would be converted to a $2 \mathrm{D}$ problem simply by changing nzoney to 1 , thus yielding a 32 by 16 rectangular regime with grid cells measuring 1 meter by 1 meter. If the code is to be run in OD (i.e., a reaction path calculation), then nzonex, nzoney, and nzonez are all set to 0 . This gives $\mathrm{nx}, \mathrm{ny}$, and $\mathrm{nz}$ all equal to 1 .

$$
\text { nzonex, }(\operatorname{nvx}(i), \operatorname{dxx}(i), i=1 \ldots \text { nzonex })
$$

1

$$
321.0
$$

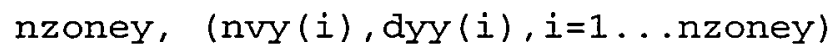

$0 \quad 16 \quad 1.00$

$$
\text { nzonez, (nvz(i), dzz(i), } i=1 \ldots \text { nzonez) }
$$

$\begin{array}{lll}0 & 5 & 0.5\end{array}$

- Card (12) NZONEX, NVX(I), DXX(I) NZONEX: number of zones in X direction with different grid spacing. 
NVX(I): number of grid cells using the grid spacing.

DXX(I): grid spacing

- Card (13) NZONEY, NVY(I), DYY(I) NZONEY: number of zones in Y direction with different grid spacing.

NVY(I): number of grid cells using the grid spacing.

DYY(I): grid spacing in Y direction.

- Card (14) NZONEZ, NVZ(I), DZZ(I)

NZONEZ: number of zones in $\mathrm{z}$ direction with different grid spacing.

NVZ(I): number of grid cells using the grid spacing.

DZZ(I): grid spacing

An example in which uneven spacing is used in the $\mathrm{X}$ direction and even spacing in the $\mathrm{Y}$ direction might be:

nzonex, (nvx(i), $\operatorname{dxx}(i), i=1 \ldots$ nzonex)

2

$\begin{array}{llll}16 & 0.5 & 16 & 2.0\end{array}$

nzoney, (nvy(i), dyy(i), $i=1 \ldots$ nzoney)

1

$16 \quad 1.00$

nzonez, (nvz(i), dzz(i), $i=1 \ldots$ nzonez)

0

$5 \quad 0.5$

In this case we have one zone in the $\mathrm{X}$ direction with spacings of 0.5 meters using 16 grid cells and a second zone (again using 16 grid cells) with a spacing of 2 meters. The grid spacing in the $\mathrm{Y}$ direction is a uniform 1 meter.

\subsection{Geochemical Conditions}

In Card (15), the code reads information on the distinct geochemical conditions to be specified in the problem. Without indicating yet where these conditions will be located (they may be initial conditions or boundary conditions), these represent the conditions which are geochemically distinct. These include information on the constraints to be used in initializing the concentrations and initial mineral volume percentages and surface areas. The list includes information on all of the primary species and minerals specified above in the input file threedin (the code checks to make sure the lists match the list in Card 8). In this case, the code will not look for an asterisk to terminate the read since it is expecting ncomp lines with information on the primary species and nrct lines on the minerals. This part of the input file takes the form

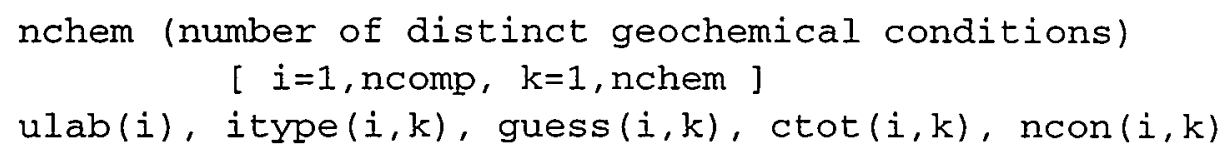


2

\begin{tabular}{|c|c|c|c|c|}
\hline$' \mathrm{cot} 2^{\prime}$ & 1 & $1 . e-10$ & $1 . e-10$ & ' ' \\
\hline 'tracer' & 1 & $1.0 e-10$ & $1.0 e-10$ & , ' \\
\hline ' $\mathrm{h}+$ ' & 7 & $6.0 e-9$ & $6 . e-9$ & ' ' \\
\hline 'fe+3' & 3 & $1.0 \mathrm{E}-15$ & $1 . e-6$ & 'goethite' \\
\hline 'al+3' & 3 & $1.0 e-20$ & $1 \cdot e-4$ & 'kaolinite' \\
\hline 'sio2 (aq)' & 3 & $1.0 e-4$ & $1 . e-4$ & 'quartz' \\
\hline 'nat' & 3 & $1.5 e-1$ & $1.5 e-1$ & 'albite' \\
\hline 'cl-' & 2 & $1.5 e-1$ & 1. $5 e-1$ & ' ' \\
\hline 'hco3-' & 4 & $1.0 e-5$ & $3.16 e-4$ & 'co2 (g)' \\
\hline '>feoh' & 5 & $3 \cdot e-6$ & 10.0 & 'goethite' \\
\hline 'goethite' & 0.001 & 100.0 & & \\
\hline 'quartz' & 0.25 & 10.0 & & \\
\hline 'gibbsite' & 0.00 & 10.0 & & \\
\hline 'kaolinite' & 0.00 & 10.0 & & \\
\hline 'albite' & 0.65 & 10.0 & & \\
\hline$' \cot 2 '$ & 1 & $1 . e-05$ & $1 . e-05$ & , ' \\
\hline 'tracer' & 1 & 0.001 & 0.001 & ' ' \\
\hline$' h+'$ & 7 & $1.0 e-5$ & $1 . e-5$ & ; ' \\
\hline fe+3' & 1 & $1 \cdot e-15$ & $1 . e-8$ &, \\
\hline$' a 1+3^{\prime}$ & 1 & $3.0 e-10$ & $1 . e-9$ & ' , \\
\hline ' sio2 (aq)' & 1 & $1.0 e-8$ & $1 . e-8$ & , ' \\
\hline 'nat' & 1 & $1.0 e-8$ & $1 . e-8$ & ' ' \\
\hline ' $\mathrm{cl}-'$ & 2 & $1.0 e-5$ & $1 . e-5$ & , ' \\
\hline 'hco3-' & 4 & $4.0 e-7$ & $3.16 e-4$ & ' $\operatorname{co2}(\mathrm{g})$ ' \\
\hline '>feoh' & 5 & $3 \cdot e-6$ & 10.0 & 'goethite' \\
\hline 'goethite' & 0.001 & 100.0 & & \\
\hline 'quartz' & 0.25 & 10.0 & & \\
\hline 'gibbsite' & 0.00 & 10.0 & & \\
\hline 'kaolinite' & 0.00 & 10.0 & & \\
\hline 'albite' & 0.65 & 10.0 & & \\
\hline
\end{tabular}

The input includes the following fields:

- Card (15) NCHEM: number of geochemically distinct initial and/or boundary conditions to be included. In what follows, the subscript $K$ refers to the geochemical condition number, while I refers to the primary species.

- NAMC(I), ITYPE(I,K), GUESS(I,K), CTOT(I,K), NCON(I,K):

NAMC(I): name of primary species which must match that specified in Card 8 .

ITYPE(I,K): constraint to be used in initialization.

1 - mass balance using total concentration, ctot.

2 - charge balance using the named species.

3 - mineral (specified by ncon) equilibrium constraint.

4 - pressure of gas (bars) fixed by $\operatorname{ctot}(j, i)$. 
5 - species is a surface complex developed on the mineral specified by ncon. In this special case, guess and ctot taken on different meanings. Guess in the case of a surface complex gives the site density on the mineral in units of moles surface hydroxyls per $\mathrm{m}^{2}$ mineral. Ctot gives the intrinsic surface area of the mineral to be used in computing an initial total number of surface hydroxyl sites per mass solution (units of $\mathrm{m}^{2} \mathrm{~g}^{-1}$ ).

7 - activity of species is fixed by guess $(j, i)$.

8 - concentration of species is fixed by guess $(j, i)$.

GUESS(I,K): initial guess for species concentration. In the case of options 7 or 8 , guess actually fixes either the concentration or the activity of the species. In the case of option 5 (primary species is a surface complex), guess is the moles surface hydroxyls per $\mathrm{m}^{2}$ mineral.

CTOT(I,K): total concentration of species if $i$ type $=1$. If $i$ type $=4$ (gas constraint), ctot is used to specify the partial pressure of the gas (e.g., $\mathrm{O}_{2(g)}$ ), while guess is used as a guess of the concentration of the basis species related to the gas (e.g., $\mathrm{CO}_{2(a q)}$ ). If option 5 (primary species is a surface complex) is specified, then ctot is the intrinsic surface area of the mineral in units of $\mathrm{m}^{2} \mathrm{~g}^{-1}$ which will be used in computing a total (initial) concentration of the surface hydroxyl.

$\operatorname{NCON}(\mathrm{I}, \mathrm{K})$ : name of mineral (if itype $=3$ ) or gas (if $i$ type $=4$ ) (enclosed in single quotes) to be used as equilibrium constraint for named species. If i type $=5$, then this is the mineral upon which the surface hydroxyl sites are developed. This ensures that the total number of adsorption sites, which otherwise can be treated similarly to other total concentrations in the system, is linked to the potentially changing surface area of the mineral.

- $\operatorname{NAMK}(\mathrm{I}), \operatorname{VOLIN}(\mathrm{I}, \mathrm{K}), \operatorname{AREAIN}(\mathrm{I}, \mathrm{K})$

NAMK(I): character string containing mineral name.

VOLIN $(\mathrm{I}, \mathrm{K})$ : initial volume fraction of mineral $I$ in the $K$ th zone.

$\operatorname{AREAIN}(\mathrm{I}, \mathrm{K})$ : initial reactive surface area of mineral $\mathrm{I}$ in the $\mathrm{K}$ th zone. Units of $\mathrm{m}_{\text {mineral }}^{2}$ $\mathrm{m}_{\text {rock }}^{-3}$.

\subsection{Spatial Distribution of Geochemical Conditions}

Information on the spatial distribution of geochemical conditions is read in Cards 16 and 17. The code assumes that geochemical condition 1 is the default and will initialize the entire interior domain with these concentrations and constraints if no other internal zones are specified in Card 16. The internal domains are assumed to be orthogonal blocks (which may have dimensions of zero width). In the example which follows, one internal zone is specified which begins at $\mathrm{JX}=1$ and ends at $\mathrm{JX}=3$ (inclusive). This example could apply either to a $1 \mathrm{D}$ problem (where we automatically assume $\mathrm{NY}=1$, or it could be a $2 \mathrm{D}$ problem where we are initializing a strip 3 grid cells along $J Y=1$. The code will read up to 100 internal zones (i.e., the number of heterogeneities) and will terminate only when it finds a geochemical condition equal to 0 (the geochemical condition 0 in this respect is similar to " $*$ " used above to terminate character string reads).

This section can also be used to specify the kind of boundary condition and/or initial condition desired, in addition to the geochemical condition to be used. In the case of internal zones specified in Card 16, a 
positive geochemical condition number indicates that the rectangular domain which is specified should be initialized to the appropriate geochemical condition ( 2 in the example below), but that the concentrations in this zone will not remain fixed once the transient calculation begins. In contrast, if a negative geochemical condition is specified ( -2 in the example below), the code will fix the concentrations to those specified by the appropriate geochemical condition number (i.e., 2 in this case) for the duration of the transient calculation. As discussed in the section on Initial and Boundary Conditions, this option is useful particularly when using the code OS3d if one wants to specify a Dirichlet boundary (fixed concentration boundary). We do so by fixing the first interior node in from the boundary, thus effectively sliding the location of the boundary in one grid cell. This is not necessary to do where the flow of fluid is into the interior domain at this point, since in this case both OS3D and GIMRT will grab the upstream, exterior node with the appropriate geochemical condition specified in Card 17.

In Card 17, 6 different condition numbers must be provided, even in OD cases since the code will expect to read these. The geochemical conditions are those to be specified along an entire face of the domain (i.e., we visualize the internal domain as cubic). Negative and positive geochemical condition numbers play a similar role to the roles they play in Card 16, except that the two codes behave somewhat differently here. If one is using GIMRT, then specifying a negative geochemical condition number implies a Dirichlet condition. This is unnecessary where the flow is entering the domain along this face, since in this case the code will automatically use the upstream, exterior node in computing the advective flux across the face. But where the advective flux is zero, specifying a negative geochemical number turns the boundary condition along this face from a no-flux condition into a Dirichlet condition. This is not possible in OS3D at the present time because of the way the transport algorithm is set up, however. As noted above, if Dirichlet conditions are desired in the case of OS3D, internal nodes immediately adjacent to the boundary should be used with negative numbers. This method also has the advantage that one may specify individual nodes rather than forcing an entire face to have a single type of boundary condition.

INTERNAL spatially distinct zones OVER AND ABOVE default $k$ (condition \#), $j x, j y, j z$ (lower left), jx,jy,jz (upper right) $j \mathrm{x}=\mathrm{x}$ direction, $j \mathrm{y}=\mathrm{y}$ direction, $\mathrm{jz}=\mathrm{z}$ direction 


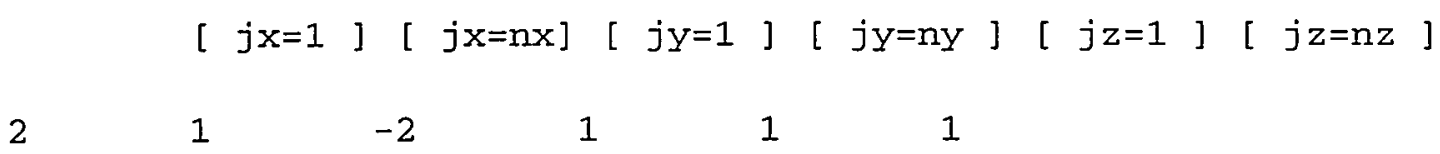

In the first example, geochemical condition 2 is used along the left hand face of the domain (i.e., JX = 1), while geochemical condition 1 is used at all other faces. However, only those faces which have flow entering the domain from outside or those which specify a negative geochemical condition number (none in the first example) will actually influence the interior domain. In the second example, we fix grid cell 1 to be geochemical condition 2 for the entire duration of the transient calculation, thus effectively turning it into a Dirichlet boundary condition at grid cell 1 . This would be redundant, however, if the flow were coming into the domain at this point in the $\mathrm{X}$ direction, since Card 17 tells the code to use geochemical condition 2 in computing the advective flux across the left hand face $(J X=1)$. Note that we have specified at the face corresponding to JY $=1$ that geochemical condition 2 to be used and that it be treated as a Dirichlet condition. Only GIMRT will actually do anything with this. If this option is tried in OS3D, the code will respond by suggesting that the user fix an interior node using Card 16.

- Card (16) NDIST(I), JXXLO(I),JXXHI(I),JYYLO(I),JYYHI(I),JZZLO(I),JZZHI(I):

NDIST(I): geochemical condition number (according to the order given in Card 15) to be distributed in space. If the geochemical number is negative (see second example above), the code will treat this domain as fixed throughout the simulation. If the geochemical condition is positive, then the code will initialize the domain to the appropriate geochemical condition, but these will not remain fixed as transport and reactions act on the grid cells. The geochemical condition 1 is redundant since in the absence of information here, the code will use condition 1 as the default, but it is allowed nontheless. This section is terminated when the code encounters a value for ndist $=0$ (this must be there for a correct read).

JXXLO(I): grid point in X direction specifying lower left hand corner of a rectangular domain to be initialized with geochemical condition ndist.

JXXHI(I): grid point in X direction specifying upper right hand corner of a rectangular domain to be initialized with geochemical condition ndist.

JYYLO(I): grid point in Y direction specifying lower left hand corner of a rectangular domain to be initialized with geochemical condition ndist.

JYYHI(I): grid point in Y direction specifying upper right hand corner of a rectangular domain to be initialized with geochemical condition ndist.

JZZLO(I): grid point in Z direction specifying lower left hand corner of a rectangular domain to be initialized with geochemical condition ndist.

JZZHI(I): grid point in $\mathrm{Z}$ direction specifying upper right hand corner of a rectangular domain to be initialized with geochemical condition ndist.

- Card (17) JBD(I): geochemical condition to be used at the boundaries of the 3D rectangular volume. The code expects to read 6 numbers here, one for each edge of the cube, even for $2 D, I D$, and $O D$ problems. A negative geochemical condition number is used only by GIMRT, transforming what would otherwise be a no-flux boundary into a Dirichlet boundary (see section on Initial and Boundary Conditions.) 


\subsection{Reaction Path Switch and Plotting Information}

Card 18 specifies whether the code should be run in reaction path mode or batch mode if no discretization is being used (i.e., $n x, n y$, and $n z=1$ ). In reaction path mode, the mineral volume fractions are not updated since we assume that all reaction products are left behind. Card 19 contains information about plotting species concentrations at specific points in space. In the example given below, Card 19 tells the code to write to the datafile breakthrough. out the species $\mathrm{H}^{+}$and $\mathrm{HCO}_{3}^{-}$at grid point 1,1,1 every 2 timesteps.

CHOOSE BATCH OR REACTION PATH (ONLY APPLIED WHERE NXYZ = 1)

(ipath $=1$ for reaction path, ipath $=0$ for batch)

1

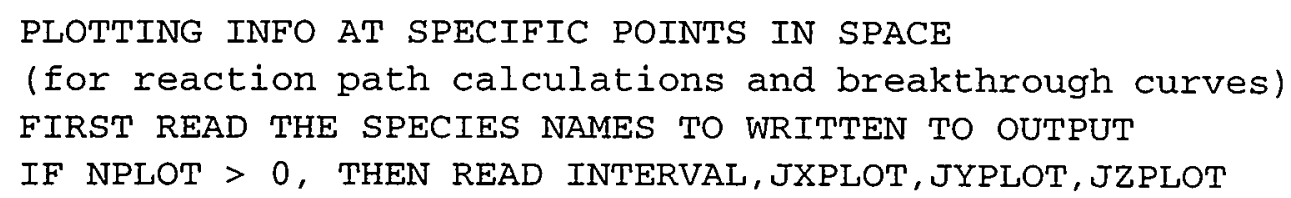

2

$1 \quad 1 \quad 1$

- Card (18) IPATH: parameter only used when nxyz = 1 (i.e., no spatial discretization) and controlling whether the mineral volume fractions are updated. When ipath $=1$ (and nxyz $=1$ ), the code runs in reaction path mode in order to model the movement of a fluid packet through the rock, leaving reaction products behind. When ipath $=0$, the code runs in true batch mode and the reaction products remain in the closed system.

- Card (19) PLOTDUMMY(I): character string for species to be plotted at a specific point in space.

- ' $*$ ' marks end of read.

- INTERVAL, JXPLOT, JYPLOT, JZPLOT: interval at which time-dependent data (the species listed above in plotdummy) will be written to the output file breakthrough. out, followed by the location in $\mathrm{X}, \mathrm{Y}$, and $\mathrm{Z}$ space of the point of interest. For a reaction path calculation, jxplot, jyplot, and jzplot should be all equal to 1 .

\subsection{Transport Parameters}

In the last section of the input file threedin, the code reads in Cards 20 through 22 information about the transport parameters to be used in the problem. The velocities specified are Darcy velocities in units of $\mathrm{m}^{3} / \mathrm{m}^{2} / \mathrm{yr}$. The user has the option of specifying either a constant velocity in a particular coordinate direction or to give 3 separate filenames where the code can read the $\mathrm{X}, \mathrm{Y}$, and $\mathrm{Z}$ components of the velocity 
field. Note that the code will attempt to read 3 character strings for the velocity files even if the parameter ivelocity is set to 0 . In the same way, the code will read the components of the constant velocity field even if this information is not going to be used. After this information is read, the code reads the value of the molecular diffusion coefficient to be used. A sample input follows:

VELOCITY FIELD OPTIONS ( $0=$ CONSTANT VELOCITIES, $1=$ FILE READ) (in the order $Q X, Q Y, Q Z$ )

0

$0.5 \quad 0.0 \quad 0.0$

'custom $32 \times 16 . v x$ ' custom $32 \times 16 . v y '$ 'custom $32 \times 16 . v z$ '

HYDRODYNAMIC DISPERSION

$0.000000001 \quad 0.000000001$

(22) MOLECULAR DIFFUSION

idiffus, dcoeff, porpow

$1 \quad 1 . d-09 \quad 1.0$

- Card (20) IVELOCITY: flag indicating whether to use a constant velocity field or to use the information found in the files specified below.

0 - assume constant velocities given in qxinit, qyinit, and qzinit below

1 - use velocity components found in the files listed below.

- QXINIT, QYINIT, QZINIT: X, Y, and Z components of the constant velocity field. Code will attempt to read these numbers even if ivelocity $=1$, but the information will not be used.

- VXFILE, VYFILE, VZFILE: character strings containing file names with velocity components. This field should include 3 character strings, even if the code is to be run with a constant velocity, otherwise a read error will result. In the files, since the velocities are face-centered, the $X$ component of the velocity should have dimensions of $(0: n x, 1: n y, 1: n z)$, the $Y$ component (1:nx,0:ny, $1: n z)$, and the $Z$ component (1:nx,1:ny, $0: n z)$.

- Card (21) ALFL, ALFT: longitudinal and transverse dispersivities (in meters) respectively.

- IDIFFUS, DCOEFF, PORPOW

IDIFFUS: flag controlling choice of a diffusion coefficient.

0 - default, $1 \times 10^{-9} \mathrm{~m}^{2} \mathrm{~s}^{-1}$ and an activation energy of $5 \mathrm{kcal} / \mathrm{mole}$.

1 - user provided (uses dcoeff).

DCOEFF: user provided diffusion coefficient, $D_{0}$, in units of $\mathrm{m}^{2} \mathrm{~s}^{-1}$ (only used if idif fus $=1$ ).

PORPOW: the cementation exponent in Archie's Law (Equation 5). 


\section{PARAMETERS IN FORTRAN FILES}

Some of the parameters which the user may want to change are located within the FORTRAN source files themselves. These are normally parameters which may not require change as commonly as those in threedin. Note, however, that if any of these parameters within the source files are changed, the code must be recompiled with the make command.

The code GIMRT uses the WATSOLV software package (VanderKwaak et al., 1995) to solve the global system of equations. The code OS3D uses the package to solve the dispersion/diffusion equation. The various values of these parameters which may be used are described in detail in the WATSOLV manual, but they are reviewed briefly here. The data statement at the top of the files gimrt. $f$ and newton.f take the form

C

watsolv input parameters

data level/1/, idetail/0/,rtwotol/1.e-25/, rmaxtol/1.e-25/,

\&smaxtol/1.e-12/,nitmax/100/, solve_flag/.false./,north/2/

The parameters have the following meaning:

level factorization level (number of grid connections);

idetail solver performance detail level

0 -no information,

1 -summary information,

2-full information;

rtwotol residual Euclidean (two) norm convergence (error) criteria;

rmaxtol residual infinity (maximum norm convergence (error) criteria;

smaxtol solution update infinity (maximum) norm scale criteria;

nitmax maximum number of iterations to perform;

solve_flag logical flag to indicate acceleration method

true.-GMRES acceleration,

false.-CGSTAB acceleration;

north number of basis vectors to retain in GMRES.

Other input parameters for GIMRT are also listed in data statements:

startup parameters
data nend/5000000/, newton/50/cormax/5.0/, atol/1.e-09/, rtol/1.e-07/

These parameters are defined as:

nend total number of allowable timesteps;

newton maximum number of Newton iterations;

corrmax maximum correction in log space for Newton update;

atol absolute tolerance for convergence on residuals $\left(\mathrm{mol} / \mathrm{m}^{3}\right)$;

rtol relative tolerance for convergence on residuals $\left(\mathrm{mol} / \mathrm{m}^{3}\right)$;

For OS3D, there are some additional parameters set in the driver routine os $3 \mathrm{~d}$. $\mathrm{f}$, the most important of which are logical flags choosing whether the code should be run with sequential iteration or straight operator splitting (i.e., sequential coupling). These include 


$$
\begin{aligned}
& \text { data nend/5000000/,picard/.false./, } \\
& \text { \& picardtol/1.e-10/,omeg/0.50/ }
\end{aligned}
$$

where

nend total number of allowable timesteps;

picard logical flag indicating whether to use sequential iteration or coupling, false.-use classic operator splitting or sequential coupling, true.-use sequential iteration;

picardtol tolerance $(\mathrm{mol} / \mathrm{kg})$ on convergence of sequential iteration; omeg damping of sequential iteration to improve convergence.

We recommend the use of omeg equal to 0.5 or the sequential iteration method does not converge particularly well. In the file newton. $f$, the user will find parameters similar to those listed under gimrt. $f$ above controlling the number of allowable Newton iterations and the convergence tolerances.

\section{OUTPUT IN THE FILE THREEDOUT}

Upon completion of the initialization procedure for either OS3D or GIMRT, information about the initialization, the stoichiometric reaction matrices (i.e., how the reactions are actually written), the $\log \mathrm{Ks}$ and a host of other parameters is written out to the file threedout. The first part of the file summarizes the various control parameters used in the run. The next part lists the primary and secondary species, the minerals, and the gases chosen for the run. The code then writes out the stoichiometric coefficient matrix and the log Ks for the secondary species, minerals, and gases. The user would do well to examine this matrix, since it represents in tableau form the reactions written in terms of the user-provided primary species. The code has also transformed the log Ks in the EQ3 database into the appropriate log $\mathrm{K}$ for the reaction as written given the user-provided primary species. Following this is information on the mineral rate laws, basically summarizing what the user provided in Card 10 of threedin. Next is the information on the discretization and the geochemical conditions provided (at this point just regurgitation of the user-provided input). Below this section is information on the surface complexation, including the calculated total concentrations of surface hydroxyls on the various minerals in the system. Then the code proceeds to the actual initialization routine and provides full speciation given the constraints provided by the user. This section includes information on the ionic strength, the $\mathrm{pH}$, the charge balance, and the concentration of the individual (primary and secondary) species after the convergence of the initialization routine. At the end is a summary of the transport parameters which will be used in the run.

\section{GRAPHICS OUTPUT}

The subroutine graphics. $f$ creates a number of files which write out the values of the important quantities (e.g., $\mathrm{pH}$, reaction rate) as a function of space at specified times during the course of the simulation. Up to 19 files representing 19 different times may be created during any one simulation (e.g, ph1 . out ... ph19. out). The files include

- master1. out: writes the concentration of the "master" variable (specified in threedin. If the species is $\mathrm{H}^{+}$, the code writes the solution $\mathrm{pH}$. Otherwise, the logarithm of the concentration is written. 
- conc1. out: writes all of the linear concentrations.

- rate1 . out: writes the reaction rates of the minerals in units of moles liter bulk volume $\mathrm{e}^{-1} \mathrm{sec}^{-1}$. The user may change the units to volume $\% \mathrm{yr}^{-1}$ by uncommenting and commenting the appropriate lines in the graphics output files graph1d.f, graph2d.f, and graph3d.f.

- volume1.out: writes the volume \% of the minerals and the porosity.

- satur1. out: writes the saturation indices of the minerals $(\log \mathrm{Q} / \mathrm{K})$.

The format of the output files may be easily modified by the user.

\section{PROBLEMS IN RUNNING THE CODE}

With a code as complicated as OS3D and GIMRT there are numerous things which could cause it to fail. Potential users who want a seamless black box which solves their problems for them automatically are urged to consider a field other than reactive transport in porous media. With some patience, however, it should be possible to figure out the quirks of these codes and also the art of handling the highly nonlinear problems which arise in reactive transport.

If the code fails during the initialization process, it is usually because of an error in the input file threedin. It is a wise policy to keep a template of the input file handy which has not been corrupted so the user may remind themselves of the structure. A common pitfall is to simply have the wrong format in the input file. The file is set up so that many of the fields are expected even where they are not used. This minimizes to some extent the changes in the actual format. So, for example as described above, the code will try to read 3 character strings containing the velocity fields even where the user has specified that either there is no advective flux or where the flow velocity is constant. These files need not exist in this case, however, since the code will not try to open them unless the parameter ivelocity described above is set to 1 .

Another common problem is a misspelling of a species name. The user may check the databases master25.data and mastertemp.data and any other they have generated to verify that their spelling is correct. If the message appears that a species has not been found in the data base, then either the species has been misspelled or a species has been listed which is not accompanied somewhere else in the list by the appropriate species used in writing the reaction in the EQ3 data base. This will occur, for example, if one were to include redox reactions without listing $\mathrm{O}_{2(g)}$ among the gases in Card 11. One would also get an error message if one lists a species for which the necessary basis species are missing in the input file.

It is also fairly common to have problems in obtaining convergence during the initialization process. This usually occurs when a mineral constraint is specified and the initial guess for the primary species concentrations is off by many orders of magnitude. This is common in particular with redox species. At this writing, the initialization procedure in both OS3D and GIMRT rely on a damped Newton-Raphson method, which in future developments should be accompanied by a somewhat more robust method where the initial guess may or may not be good or is unnecessary altogether. In certain other cases, the initialization may fail to converge due to nonsensical input, as for example where the specified total concentrations and mineral constraints would make charge balancing impossible. If convergence problems during the initialization are encountered, it is usually advisable to replace the charge balance and mineral constraints with constraints on the total concentrations so that one can gradually improve the initial guesses for the basis species. 
If non-convergence occurs as the code steps through time, one can reduce the value of ttol in the input file threedin. This parameter controls the rate at which the time step is increased based on the time truncation error. In addition, one can specify a smaller value for the maximum timestep tstep. If the code blows up during the first few time steps, then try reducing the size of the initial time step. Once the system relaxes, the time step will be gradually increased. Non-convergence during the time stepping routine is generally more likely as the reaction rates are increased toward the local equilibrium limit. Large reaction rate constants may require the use of smaller time steps. If necessary, one may also try a different value for the parameter bpeg if basis switching is used. Bpeg may be changed in the input file threedin.

\section{CUSTOMIZING THE CODE}

There are a number of changes which can be made either to the code or to the data bases to customize GIMRT and OS3D for a particular problem.

\subsection{Array Dimensions}

As mentioned above, it may be necessary or advisable to change the dimensions used in the code which are specified in the file params. inc and paramsos. inc. If this file is changed, GIMRT and OS3D must be recompiled with the make command. The code checks to make sure that the array dimensions are large enough, but it will not, of course, scale the dimensions back for smaller problems. The dimension of the arrays may be an issue if GIMRT in particular is being run on a machine without a fair amount of physical memory (> 16 megabytes).

\subsection{Modifying the Data Base}

The data base read by GIMRT and OS3D is divided into four blocks. The first block is a list of basis species not included on the left hand side of a chemical reaction with the $a_{0}$ radius parameter used in the Debye-Hückel equation, the species valence, and the molecular weight of the species. The second block contains chemical reactions and thermodynamic $\log K$ data for aqueous species, the third block mineral data, and the fourth block data for gases. The various blocks are separated by lines beginning with the string 'null'. The first line of the data base contains the temperature for each $\log K$ entry with the total number of values.

First line: 'temp.' $N T_{1} T_{2} \ldots T_{N}$.

Format for basis species: <'component name' $><$ ion size $><$ charge $><$ molecular weight $>$

Format for aqueous complexes: <'species name' $><\#$ of component species in reaction $>$ $<$ stoichiometric coeff. $><$ 'component name' $>\cdots<\operatorname{logK}$ 's $N$-values $><$ ion size $>$ $<$ charge $><$ molecular weight $><$ 'comment'>

Format for minerals: <'species name' $><$ molar volume $><\#$ of component species in reaction $>$ $<$ stoichiometric coeff. $><$ 'component name' $>\cdots<\operatorname{logK}$ 's $N$-values $><$ molecular weight $>$ <'comment'> 
Format for gases: <'species name' $><$ molar volume $><$ \# of component species in reaction $>$ $<$ stoichiometric coeff. $><$ 'component name'> $\cdots<\operatorname{logK}$ 's $N$-values $><$ molecular weight $>$ $<$ 'comment'>

The sign of the $\log K$ must conform to the reaction written with the aqueous complex, mineral or gas on the left hand side, that is, as the destruction of one mole of the aqueous complex, mineral, or gas. A sample data base is shown in Table 12.5. The data base delivered with the code is a transformed version of the EQ3/6 data base created by Peter Lichtner at Southwest Research Institute. This data base contains over 1500 aqueous species and minerals. Log $K$ values are stored at temperatures of $0,25,60,100,150$, 200,250 and $300^{\circ} \mathrm{C}$ and interpolated according to the expression

$$
\log K=\frac{a_{-2}}{T^{2}}+\frac{a_{-1}}{T}+a_{0} \ln T+a_{1}+a_{2} T
$$

where $T$ denotes the absolute temperature in degrees Kelvin. Pressure lies along the steam saturation curve. Other data bases may be generated using the computer code SUPCRT (Johnson et al., 1992) for any desired temperature range and pressure.

\subsection{Adding or Modifying Kinetic Rate Laws}

The kinetic rate laws presently implemented in GIMRT and OS3D all include some kind of saturation dependence of the rate. This ensures that the rates go to zero when equilibrium is reached. Currently the codes assume the rate law prevails during precipitation, although one can specify obtain some differences in the dissolution and precipitation rate by specifying dependences on the dissolution rate far from equilibrium. Josep Soler of Yale University recently added the capability for differing precipitation and dissolution rate laws to an earlier version of the code, so this capability may be added to next release of the code. These expressions can also handle nonlinear expressions by adjusting the parameters $n$ and $\mathcal{M}$ in Equation 28. By providing dependences on the dissolution rates far from equilibrium (where most experimental studies to date have been carried out), one can investigate a range of reaction mechanisms, including $\mathrm{pH}$ dependent and $\mathrm{pH}$ independent rates, a dependence on the chlorinity of the solution, and even ligand-promoted dissolution (i.e., it is possible to provide a dependence on a surface complex. The rate law may depend on either secondary or primary species. One can add other rate expressions, however, by altering the source code. This involves adding or substituting the appropriate function to the overall rate for a mineral computed in the subroutine reaction. $f$. In addition, one must add the derivative of this function with respect to the various basis species to the Jacobian calculation in the subroutine rctder. $f$.

\subsection{Providing and/or Updating a Velocity Field}

As described above in the sections describing the input file threedin, the user may specify that a velocity file should be read in. Note that since the velocities are needed at the faces of grid cells, the X components of the velocity must go from (0:nx, $1: n y, 1: n z)$, the Y component from (1:nx,0:ny, $1: n z)$, and the $\mathrm{Z}$ component from (1:nx, 1,ny,0:nz). The velocities are to be stored so that they can be read with an implicit do loop of the form (for a 3D problem)

$$
\begin{array}{lll}
\operatorname{read}\left(23,{ }^{*}\right) & (((q x(j x, j y, j z), j x=0, n x), j y=1, n y), j z=1, n z) \\
\operatorname{read}(24, *) & (((q y(j x, j y, j z), j x=1, n x), j y=0, n y), j z=1, n z) \\
\operatorname{read}\left(25,{ }^{*}\right) & (((q z(j x, j y, j z), j x=1, n x), j y=1, n y), j z=0, n z)
\end{array}
$$


or as

$$
\begin{aligned}
& \operatorname{read}(23, *) \quad(((q x(j x, j y, j z), j x=0, n x), j y=1, n y), j z=1, n z) \\
& \operatorname{read}(24, *) \quad(((q y(j x, j y, j z), j x=1, n x), j y=0, n y), j z=1, n z)
\end{aligned}
$$

for a $2 \mathrm{D}$ problem (i.e., where $j z=1$ ).

The position in the driver routines os $3 \mathrm{~d} . \mathrm{f}$ and gimrt. $\mathrm{f}$ to place an update of a transient velocity field is marked by

C PLACE ROUTINE TO UPDATE TRANSIENT VELOCITY FIELD HERE

\subsection{Adding a Temperature Field}

The appropriate place within the startup routine for GIMRT (startup_gi . f) to place a steady-state calculation of the temperature field is marked by the lines

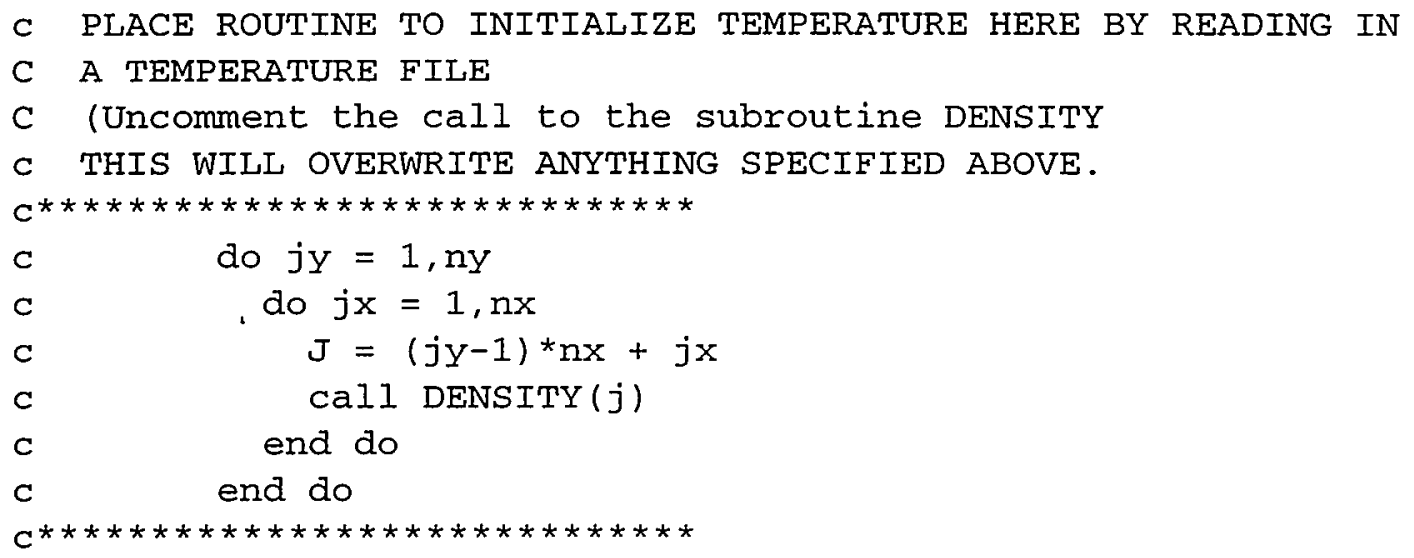

If one wishes to include a transient calculation of the temperature field, the position in gimrt. $f$ inside the time loop is indicated by the lines

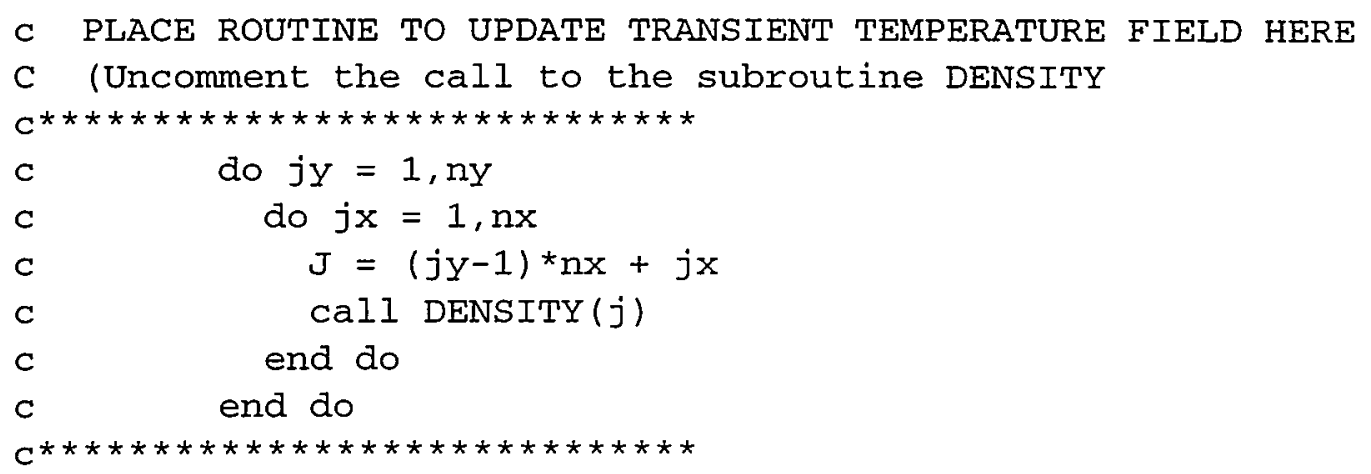

The temperature field is block-centered (unlike the face-centered temperatures), so the temperature will have dimensions of (1:nx,1:ny, $1: n z)$. 
Table 1: Sample data base read by GIMRT and OS3D for a single temperature of $25^{\circ} \mathrm{C}$.

$\begin{array}{lrll}\text { 'temp.' } 125 . & & \\ \text { 'h2o' } & 3.0 & 0.0 & 18.01528 \\ \text { 'al+3' } & 9.0 & 3.0 & 26.98154 \\ \text { 'h+' } & 9.0 & 1.0 & 1.00794 \\ \text { 'hco3-' } & 4.0 & -1.0 & 61.01714 \\ \text { 'sio2(aq)' } & 3.0 & 0.0 & 60.08430 \\ \text { 'null' } & 0 . & 0 . & 0.0\end{array}$

'al (oh) 2+' $3-2.0$ 'h+' 1.0 'al+3' 2.0 'h2o' 10.09914 .001 .0060 .99622

'al (oh) $3(\mathrm{aq})$ ' $3-3.0$ ' $\mathrm{h}+$ ' $^{\prime} 1.0$ 'al+3' 3.0 'h2o' 16.1577

$3.00 \quad 0.0078 .00356$

'al (oh) 4-' $3-4.0$ ' $h+$ ' 1.0 'al+3' 4.0 'h20' $22.14774 .00-1.0095 .01090$

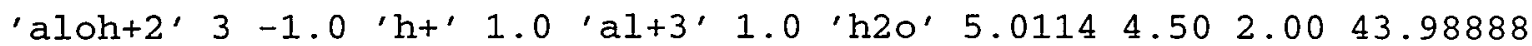

' $\mathrm{co} 2$ (aq)' $3-1.0$ 'h20' 1.0 ' $\mathrm{h}+\mathrm{t}^{\prime} 1.0$ 'hco3-' -6.34473 .000 .0044 .00980$

'co3-2' $2-1.0$ 'h+' 1.0 'hco3-' $10.32885 .00-2.0060 .00920$

'null' 1 0. '0' 0.0.0. 0 .

'gibbsite' $31.9563-3.0$ ' $h+$ ' 1.0 'al+3' 3.0 'h2o' 6.796578 .00356

'kaolinite' $99.5204-6.0$ ' $h+$ ' 2.0 'al+3' 2.0 'sio2(aq)'

5.0 'h20' 5.1007258 .16044

'chalcedony' 22.68811 .0 'sio2(aq)' -3.728160 .08430$

'quartz' 22.68811 .0 'sio2(aq)' -3.999360 .08430$

'sio2(am)' 29.011 .0 'sio2(aq)' -2.713660 .08430$

'null' $\quad 0.010 .10$ ' 10.0

' $\operatorname{co} 2(g)$ ' 24465.0 $3-1.0$ 'h20' 1.0 ' $h+1$ ' 1.0 'hco3-' -7.813644 .00980$

'null' $\quad 0 . \quad 1 \quad 0.0^{\prime} 0.0 .0 .0$ 


\section{ACKNOWLEDGMENTS}

The present versions of the codes GIMRT and OS3D were written for the Environmental Dynamics and Simulation task which is part of the Environmental Molecular Science Laboratory (EMSL) construction project at the Pacific Northwest Laboratory in Richland, Washington. The codes are at least partly based on a code originally written while the senior author was a doctoral student at Yale University studying with Professor Antonio Lasaga. The present version of the code, however, owes much to our collaboration with Peter Lichtner while at the Universität Bern in Switzerland and since then while he has been at the Southwest Research Institute. He was responsible for writing the algorithm in the subroutine database.f which reads the thermodynamic data base and rewrites the stoichiometric coefficients and log K's in terms of the user's choice of basis species. We would also like to thank J.E. VanderKwaak, P.A. Forsyth, K.T.B. MacQuarrie, and E.A. Sudicky for making their WATSOLV sparse matrix solver available to us. We are also pleased to acknowledge the support provided by the Geoscience Program of Basic Energy Sciences (Dr. William C. Luth) of the Department of Energy (DE-FG02-90-ER14153) and to the Donors of the Petroleum Research Fund, administered by the American Chemical Society, (PRF 22364-AC2), for the support of this research (all to Antonio C. Lasaga at Yale University). In addition, we would like to acknowledge the support of the the Schweizerishes Nationalfonds in the form of a grant (21-30908.91) to Peter C. Lichtner while at the Universität Bern in Switzerland. We are grateful for the financial support of Prof. Dr. Tjerk Peters at the Mineralogisch-Petrographisches Institut at the Universität Bern. Finally, we would like to thank Ashok Chilakapati of PNNL for his careful review of the manuscript. 


\section{REFERENCES}

Aagaard P. and Helgeson H.C. (1982) Thermodynamic and kinetic constraints on reaction rates among minerals and aqueous solutions, I, Theoretical considerations. Amer. J. Sci. 282, 237-285.

Aris R. (1965) Prolegomena to the rational analysis of systems of chemical reactions. Arch. Rational Mech. and Anal. 19, 81-99.

Bear J. (1972) Dynamics of Fluids in Porous Media. American Elsevier, New York, 764 p.

Bear J. (1979) Hydraulics of Groundwater. McGraw-Hill, New York, 569 p.

Berner R.A. (1980) Early Diagenesis: A Theoretical Approach. Princeton Univ. Press, Princeton, 241p.

Bowen R.M. (1968) On the stoichiometry of chemically reacting materials. Arch. Rational Mech. Anal. 29, 114-124.

Bruges E.A., Latto B., and Ray A.K. (1966) New correlations and tables of the coefficient of viscosity of water and steam up to 1000 bar and $1000^{\circ}$ C. Internat. Jour. Heat and Mass Transfer 9, 465-480.

Daus A.D. and Frind E.O. (1985) An alternating direction Galerkin technique for simulation of contaminant transport in complex groundwater systems Water Resources Res. 21, 653-664.

Dullien F.A.L. (1979) Porous Media. Academic Press, San Diego, 396 p.

Frind E.O. and Germain D. (1986) Simulation of contaminant plumes with large dispersive contrast: Evaluation of alternating direction Galerkin models. Water Resources Res. , 22, 1857-1873.

Gupta, A.D., Lake, L.W., Pope, G.A., Sephernoori, K., and King, M.J. (1991). High-resolution monotonic schemes for reservoir fluid flow simulation. In Situ., 15, 289-317.

Helgeson H.C. (1968) Evaluation of irreversible reactions in geochemical processes involving minerals and aqueous solutions-I. Thermodynamic relations. Geochim. Cosmochim. Acta 32, 853-877.

Helgeson H.C. (1979) Mass transfer among minerals and hydrothermal solutions. In Geochemistry of Hydrothermal Ore Deposits (ed. H.L. Barnes), John Wiley and Sons, New York, 568-610.

Helgeson H.C. and Kirkham D.H. (1974) Theoretical predictions of the thermodynamic behavior of aqueous electrolytes at high pressures and temperatures; I: Summary of the thermodynamic/electrostatic properties of the solvent. Amer. J. Sci. 274, 1089-1198.

Helgeson H.C., Garrels R.M., and MacKenzie F.T. (1969) Evaluation of irreversible reactions in geochemical processes involving minerals and aqueous solutions-II. Applications. Geochim. Cosmochim. Acta 33, 455-482.

Hindmarsh, A.C. (1977) Solution of block-tridiagonal systems of linear algebraic equations. UCID30150.

Hooyman G.J. (1961) On thermodynamic coupling of chemical reactions. Proc. Nat. Acad. Sci. 47, 1169-1173.

Johnson J. W., Oelkers E. H. and Helgeson H. C. (1992) SUPCRT92: A software package for calculating the standard molal thermodynamic properties of minerals, gases, aqueous species, and reactions from 1 to 5000 bars and $0^{\circ}$ to $1000^{\circ} \mathrm{C}$, Computers in Geosciences, in press. 
Kirkner D.J. and Reeves H. (1988) Multicomponent mass transport with homogeneous and heterogeneous chemical reactions: Effect of chemistry on the choice of numerical algorithm. I. Theory. Water Resources Res. 24, 1719-1729.

Lapidus L. and Pinder G.F. (1982) Numerical Solution of Partial Differential Equations in Science and Engineering, John Wiley and Sons, New York, 677 p.

Lasaga A.C. (1981) Rate laws in chemical reactions. In Kinetics of Geochemical Processes (ed. A.C. Lasaga and R.J. Kirkpatrick), Rev. Mineral. 8, 135-169.

Lasaga A.C. (1984) Chemical kinetics of water-rock interactions. J. Geophys. Res. 89, 4009-4025.

Lichtner P.C. (1985) Continuum model for simultaneous chemical reactions and mass transport in hydrothermal systems. Geochim. Cosmochim. Acta 49, 779-800.

Lichtner P.C. (1988) The quasi-stationary state approximation to coupled mass transport and fluid-rock interaction in a porous medium. Geochim. Cosmochim. Acta 52, 143-165.

Lichtner P.C. (1992) Time-space continuum description of fluid/rock interaction in permeable media. $J$. Geophys. Res. 28, 3135-3155.

Marsily G.de (1986) Quantitative Hydrogeology. Acad. Press, New York, 440 p.

Patankar S.V. (1980) Numerical Heat Transfer and Fluid Flow. Hemisphere Publ., New York, 197 p.

Patel M.K., Cross M., and Markatos N.C. (1988) An assessment of flow oriented schemes for reducing 'false diffusion'. Int. Journ. Numerical Methods in Engineering 26, p. 2279-2304.

Press W.H., Flannery B.P., Teukolsky S.A., and Vetterling W.T. (1986) Numerical Recipes: The Art of Scientific Computing. Cambridge University Press, Cambridge, $818 \mathrm{p}$.

Reed M.H. (1982) Calculation of multicomponent chemical equilibria and reaction processes in systems involving minerals, gases, and an aqueous phase. Geochim. Cosmochim. Acta 46, 513-528.

Steefel C.I. (1992) Coupled fluid flow and chemical reaction: Model development and application to water-rock interaction. Ph.D. thesis, New Haven, Connecticut, Yale University, 234 p.

Steefel C.I. and Lasaga A.C. (1990) Evolution of dissolution patterns: Permeability change due to coupled flow and reaction. In Chemical Modeling in Aqueous Systems II (ed. D.C. Melchior and R.L. Bassett), ACS Symp. Ser. No. 416, 212-225.

Steefel C.I. and Lasaga A.C. (1992) Putting transport into water-rock interaction models. Geology 20, 680-684.

Steefel C.I. and Lasaga A.C. (1994) A coupled model for transport of multiple chemical species and kinetic precipitation/dissolution reactions with application to reactive flow in single phase hydrothermal systems. American Journal of Science, 294, 529-592.

Steefel C.I. and Lichtner P.C. (1994) Diffusion and reaction in rock matrix bordering a hyperalkaline fluid-filled fracture. Geochim. Cosmochim. Acta 58, 3595-3612.

Steefel C.I. and Van Cappellen P. (1990) A new kinetic approach to modeling water-rock interaction: The role of nucleation, precursors, and Ostwald ripening. Geochim. Cosmochim. Acta 54, 26572677.

Strang, G.. (1968) On the construction and comparison of difference schemes, SIAM J. Numer. Anal. 5, 506-517. 
Valocchi, Albert J. and Malmstead, Michael (1992). Accuracy of operator splitting for advection-dispersionreaction problems. Water Resources Res. bf 28, 1471.

VanderKwaak J.E., Forsyth P.A., MacQuarrie K.T.B., and Sudicky E.A. (1995) WATSOLV Sparse Matrix Iterative Solver Package Versions 1.01. Unpublished report, Waterloo Centre for Groundwater Research, University of Waterloo, Waterloo, Ontario, Canada. 23 p.

Van Zeggeren F. and Storey S.H. (1970) The Computation of Chemical Equilibria. Cambridge University Press, Cambridge, $176 \mathrm{p}$.

Walter A.L., Frind E.O., Blowes D.W., Ptacek C.J., and Molson J.W. (1994). Modeling of multicomponent reactive transport in groundwater: 1 . Model development and evaluation. Water Resources Res. 30, 3137-3148.

Wolery T.J., Jackson K.J., Bourcier W.L., Bruton C.J., Viani B.E., Knauss K.G., and Delany J.M. (1990) Current status of the EQ3/6 software package for geochemical modeling. In Chemical Modeling in Aqueous Systems II (ed. D.C. Melchior and R.L. Bassett), ACS Symp. Ser. No. 416, 104-116.

Zysset, A., F. Stauffer, and T. Dracos. (1994). Modeling of chemically reactive groundwater transport, Water Resources Res. 30, 2217-2228. 


\section{DESCRIPTION OF THE INPUT FILE THREEDIN}

- Card (1) LTITLE Title of simulation (up to 80 characters) enclosed in single quotes.

- Card (2) DATA1 String (up to 100 characters) with location of a user-provided thermodynamic data base. If no location is provided (i.e, the code reads' '), then the code defaults to either master 25 . data if the input temperature (given below) is $25^{\circ} \mathrm{C}$ or to mastertemp. data if another temperature is given.

- Card (3) TSTEP, DELT, TTOL, IJACOB, BPEG

TSTEP: maximum time step in years allowed.

DELT: initial time step in years.

TTOL: parameter controlling the tolerance on increasing the size of the time step. Making ttol smaller decreases the rate at which the time step is increased, for the purpose of controlling either the time truncation error or for numerical stability.

IJACOB: flag controlling update of Jacobian

0 - update Jacobian every Newton iteration

$1-$ when delt $=1 / 2$ tstep, update Jacobian every 3 Newton iterations and when delt $=$ tstep, update Jacobian every 6 iterations.

BPEG: maximum change in molality in any one Newton iteration. Reducing bpeg increases the under-relaxation of the Newton step and may sometimes prevent nonconvergence, although the total number of iterations required may actually increase.

\section{- Card (4) JPOR, CONSTANTPOR, VDISSMAX, VPPTMAX}

JPOR: flag controlling update of porosity and flow

0 - no update of porosity.

1 - porosity is updated.

CONSTANTPOR: porosity of the medium for the case where the porosity is not updated (jpor $=0$ ). If jpor $=0$, the porosity is taken from constantpor rather than from the sum of the volume fractions of the minerals in the system. If jpor $=1$ (porosity updated), constantpor is ignored.

VDISSMAX: maximum rate of decrease of a mineral's volume fraction (units of $\mathrm{yr}^{-1}$ ). Can be used to limit the amount of mineral dissolution which can occur within a single time step. Useful where either the dissolving phase is very soluble or where very small amounts are present. Where very small amounts of a mineral are present, inaccurate mineral front propagation may occur where most or all of the mineral present within a grid volume dissolves in a single time step.

VPPTMAX: maximum rate of increase of a mineral's volume fraction (units of $\mathrm{yr}^{-1}$ ).

- Card (5) JTEMP, TINIT, TGRAD

JTEMP: flag controlling temperature option.

0 - isothermal.

1 - linear temperature gradient.

TINIT: temperature in ${ }^{\circ} \mathrm{C}$ at grid point 1 .

TGRAD: temperature gradient in ${ }^{\circ} \mathrm{C} \mathrm{m}^{-1}$. A positive gradient causes the temperature to increase from grid point 1 , a negative gradient causes it to decrease.

- Card (6) MASTER, ISWITCH, IGAMMA

MASTER: string (up to 18 characters) enclosed in single quotes specifying master variable (used in controlling time step).

ISWITCH: flag controlling basis switching. 
0 - no basis switching (solves for logarithms of concentrations).

1 - not allowed at this time.

2 - basis switching every Newton iteration. Improved method over earlier versions which involves solving for the linear concentrations and using relaxation to prevent overly large Newton steps or negative concentrations. Results in reduced number of cases where non-convergence occurs, especially in redox examples. Does require some extra CPU time, however.

IGAMMA: flag controlling method for computing activity coefficients.

0 - unit activity coefficients (except for $\mathrm{H} 2 \mathrm{O}$ ).

2 - extended Debye-Hückel without calculation of the derivatives of the activity coefficients with respect to the primary species.

\section{- Card (7) NSTOP, PRTINT}

NSTOP: number of graphics files to be printed out.

0 - no time stepping, distribution of species only.

$\geq 1$ - number of output files at prtint intervals.

PRTINT: times at which output should be written (at least nstop times should be listed.

\section{- Card (8) PRIMARY SPECIES}

- NAMC(J) name of primary species to be included (enclosed in single quotes).

- ' $*^{\prime} \quad$ marks end of primary species read.

- Card (9) SECONDARY SPECIES

- NAMCX(I) name (enclosed in single quotes) of the species to be included.

- ' $*^{\prime} \quad$ marks end of secondary species read.

- Card (10) NAMK(K), NREACT(K), SATI(K), SAT2(K), THRESH(K)

$\operatorname{NAMK}(\mathrm{K})$ : character string containing mineral name.

NREACT(K): number of parallel reactions involving the mineral. The code will read nreact following lines.

SAT1(K): $\quad$ order of reaction rate law ( $n$ in Equation 32).

SAT2(K): $\quad$ order of reaction rate law ( $\mathcal{M}$ in Equation 32 ).

THRESH(K): supersaturation $(\log \mathrm{Q} / \mathrm{K})$ necessary for precipitation.

- NPRE(LL,K), DUMLABEL(MM) DEPEND(MM,LL,K), RATE0(LL,K), EA(LL,K)

NPRE(LL,K): number of species affecting the far from equilibrium dissolution rate. The code will then read npre character strings (dumlabel) and exponential dependences (depend).

DUMLABEL(MM): character string containing species name affecting far from equilibrium dissolution rate.

DEPEND(MM,LL,K): exponential dependence of the far from equilibrium rate on species given in preceding dumlabel.

RATE0(LL,K): far from equilibrium mineral dissolution rate constant at $25^{\circ} \mathrm{C}$ in units of moles $\mathrm{m}^{-2}$ $\mathrm{s}^{-1}$. Can be used as the rate constant at temperature if activation energy is set $=0$.

$\mathrm{EA}(\mathrm{LL}, \mathrm{K})$ : activation energy in units of $\mathrm{kcal}^{\mathrm{mole}} \mathrm{e}^{-1}$.

- ' $*$ ' end of mineral read. 
- Card (11) NAMG(I) name (enclosed in single quotes) of the gas to be included.

-' $*^{\prime} \quad$ marks end of read.

- Card (12) NZONEX, NVX(I), DXX(I) NZONEX: number of zones in X direction with different grid spacing. NVX(I): number of grid cells using the grid spacing.

DXX(I): grid spacing

- Card (13) NZONEY, NVY(I), DYY(I) NZONEY: number of zones in Y direction with different grid spacing.

NVY(I): number of grid cells using the grid spacing.

DYY(I): grid spacing in $Y$ direction.

- Card (14) NŹONEZ, NVZ(I), DZZ(I)

NZONEZ: number of zones in $\mathrm{z}$ direction with different grid spacing.

NVZ(I): number of grid cells using the grid spacing.

DZZ(I): grid spacing

- Card (15) NCHEM: number of geochemically distinct initial and/or boundary conditions to be included. In what follows, the subscript $\mathrm{K}$ refers to the geochemical condition number, while I refers to the primary species.

- NAMC(I), ITYPE(I,K), GUESS(I,K), CTOT(I,K), NCON(I,K):

NAMC(I): name of primary species which must match that specified in Card 8.

ITYPE(I,K): constraint to be used in initialization.

1 - mass balance using total concentration, ctot.

2 - charge balance using the named species.

3 - mineral (specified by ncon) equilibrium constraint.

4 - pressure of gas (bars) fixed by $\operatorname{ctot}(j, i)$.

5 - species is a surface complex developed on the mineral specified by ncon. In this special case, guess and ctot taken on different meanings. Guess in the case of a surface complex gives the site density on the mineral in units of moles surface hydroxyls per $\mathrm{m}^{2}$ mineral. Ctot gives the intrinsic surface area of the mineral to be used in computing an initial total number of surface hydroxyl sites per mass solution (units of $\mathrm{m}^{2} \mathrm{~g}^{-1}$ ).

7 - activity of species is fixed by guess $(j, i)$.

8 - concentration of species is fixed by guess $(j, i)$.

GUESS(I,K): initial guess for species concentration. In the case of options 7 or 8 , guess actually fixes either the concentration or the activity of the species. In the case of option 5 (primary species is a surface complex), guess is the moles surface hydroxyls per $\mathrm{m}^{2} \mathrm{~min}$ eral.

CTOT $(\mathrm{I}, \mathrm{K})$ : total concentration of species if $i$ type $=1$. If $i$ type $=4$ (gas constraint), ctot is used to specify the partial pressure of the gas (e.g., $\mathrm{O}_{2(g)}$ ), while guess is used as a guess of the concentration of the basis species related to the gas (e.g., $\mathrm{CO}_{2(a q)}$ ). If option 5 (primary species is a surface complex) is specified, then ctot is the intrinsic surface area of the mineral in units of $\mathrm{m}^{2} \mathrm{~g}^{-1}$ which will be used in computing a total (initial) concentration of the surface hydroxyl.

NCON(I,K): name of mineral (if itype $=3$ ) or gas (if itype $=4$ ) (enclosed in single quotes) to be used as equilibrium constraint for named species. If itype $=5$, then this is the mineral upon which the surface hydroxyl sites are developed. This ensures that the total number of adsorption sites, which otherwise can be treated similarly to other total concentrations in the system, is linked to the potentially changing surface area of the mineral. 
- NAMK(I), VOLIN(I,K), AREAIN(I,K)

NAMK(I): character string containing mineral name.

VOLIN(I,K): initial volume fraction of mineral I in the Kth zone.

AREAIN(I,K): initial reactive surface area of mineral $I$ in the Kth zone. Units of $\mathrm{m}_{\text {mineral }}^{2} \mathrm{~m}_{\text {rock }}^{-3}$.

- Card (16) NDIST(I), JXXLO(I),JXXHI(I),JYYLO(I),JYYHI(I),JZZLO(I),JZZHI(I):

NDIST(I): geochemical condition number (according to the order given in Card 15) to be distributed in space. If the geochemical number is negative (see second example above), the code will treat this domain as fixed throughout the simulation. If the geochemical condition is positive, then the code will initialize the domain to the appropriate geochemical condition, but these will not remain fixed as transport and reactions act on the grid cells. The geochemical condition 1 is redundant since in the absence of information here, the code will use condition 1 as the default, but it is allowed nontheless. This section is terminated when the code encounters a value for ndist $=0$ (this must be there for a correct read).

JXXLO(I): grid point in X direction specifying lower left hand corner of a rectangular domain to be initialized with geochemical condition ndist.

JXXHI(I): grid point in X direction specifying upper right hand corner of a rectangular domain to be initialized with geochemical condition ndist.

JYYLO(I): grid point in Y direction specifying lower left hand corner of a rectangular domain to be initialized with geochemical condition ndist.

JYYHI(I): grid point in Y direction specifying upper right hand corner of a rectangular domain to be initialized with geochemical condition ndist.

JZZLO(I): grid point in $Z$ direction specifying lower left hand corner of a rectangular domain to be initialized with geochemical condition ndist.

JZZHI(I): grid point in $\mathrm{Z}$ direction specifying upper right hand corner of a rectangular domain to be initialized with geochemical condition ndist.

- Card (17) JBD(I): geochemical condition to be used at the boundaries of the 3D rectangular volume. The code expects to read 6 numbers here, one for each edge of the cube, even for $2 D, I D$, and $O D$ problems. A negative geochemical condition number is used only by GIMRT, transforming what would otherwise be a no-flux boundary into a Dirichlet boundary (see section on Initial and Boundary Conditions.)

- Card (18) IPATH: parameter only used when nxyz = 1 (i.e., no spatial discretization) and controlling whether the mineral volume fractions are updated. When ipath $=1$ (and $n x y z=1$ ), the code runs in reaction path mode in order to model the movement of a fluid packet through the rock, leaving reaction products behind. When ipath $=0$, the code runs in true batch mode and the reaction products remain in the closed system.

- Card (19) PLOTDUMMY(I): character string for species to be plotted at a specific point in space.

- ' $*$ ' marks end of read.

- INTERVAL, JXPLOT, JYPLOT, JZPLOT: interval at which time-dependent data (the species listed above in plotdummy) will be written to the output file breakthrough. out, followed by the location in $X, Y$, and $Z$ space of the point of interest. For a reaction path calculation, jxplot, jyplot, and $j z p l o t$ should be all equal to 1 .

- Card (20) IVELOCITY: flag indicating whether to use a constant velocity field or to use the information found in the files specified below.

0 - assume constant velocities given in qxinit, qyinit, and qzinit below

1 - use velocity components found in the files listed below. 
- QXINIT, QYINIT, QZINIT: X, Y, and Z components of the constant velocity field. Code will attempt to read these numbers even if ivelocity $=1$, but the information will not be used.

- VXFILE, VYFILE, VZFILE: character strings containing file names with velocity components. This field should include 3 character strings, even if the code is to be run with a constant velocity, otherwise a read error will result.

- Card (21) ALFL, ALFT: longitudinal and transverse dispersivities (in meters) respectively.

- IDIFFUS, DCOEFF, PORPOW

IDIFFUS: flag controlling choice of a diffusion coefficient.

0 - default, $1 \times 10^{-9} \mathrm{~m}^{2} \mathrm{~s}^{-1}$ and an activation energy of $5 \mathrm{kcal} / \mathrm{mole}$.

1 - user provided (uses dcoeff).

DCOEFF: user provided diffusion coefficient, $D_{0}$, in units of $\mathrm{m}^{2} \mathrm{~s}^{-1}$ (only used if idiffus $=1$ ). PORPOW: the cementation exponent in Archie's Law (Equation 5). 